\title{
On the regularity of critical and minimal sets of a free interface problem
}

\author{
NiCOLA FUSCO \\ Dipartimento di Matematica e Applicazioni "R. Cacciopoli”, \\ Università degli Studi di Napoli “Federico II”, Napoli, Italy \\ E-mail:n.fusco@unina.it \\ VESA JULIN \\ Department of Mathematics and Statistics, University of Jyväskylä, Finland \\ E-mail: vesa.julin@jyu.fi
}

[Received 26 May 2014 and in revised form 23 September 2014]

\begin{abstract}
We study a free interface problem of finding the optimal energy configuration for mixtures of two conducting materials with an additional perimeter penalization of the interface. We employ the regularity theory of linear elliptic equations to study the possible opening angles of Taylor cones and to give a different proof of a partial regularity result by Fan Hua Lin [15].
\end{abstract}

2010 Mathematics Subject Classification: Primary 49Q10, 49N60, 74G40

Keywords: Free interface, regularity of minimal surfaces, Taylor cones

\section{Introduction}

In this paper we consider the functional

$$
\mathcal{F}(E, v)=\gamma P(E, \Omega)+\int_{\Omega} \sigma_{E}(x)|D v|^{2} d x
$$

where $\gamma>0, \Omega \subset \mathbb{R}^{n}$ is an open set, $v \in W^{1,2}(\Omega)$ and $P(E, \Omega)$ stands for the perimeter of $E$ in $\Omega$. Moreover, $\sigma_{E}(x)=\beta \chi_{E}(x)+\alpha \chi_{\Omega \backslash E}(x)$, where $0<\alpha<\beta<\infty$ are given constants.

Given a function $u_{0} \in W^{1,2}(\Omega)$ and a measurable set $E \subset \Omega$, we denote by $u_{E}$, or simply by $u$ if no confusion arises, the corresponding elastic equilibrium, i.e., the minimizer in $W^{1,2}(\Omega)$ of the functional

$$
\int_{\Omega} \sigma_{E}(x)|D v|^{2} d x
$$

under the boundary condition $v=u_{0}$ on $\partial \Omega$. It follows that the function $u$ solves the linear equation

$$
\int_{\Omega}\left\langle\sigma_{E} D u, D \varphi\right\rangle d x=0 \quad \text { for every } \varphi \in W_{0}^{1,2}(\Omega)
$$

If we denote by $u_{\beta}$ and $u_{\alpha}$ the restriction of $u$ on $E$ and $\Omega \backslash E$, respectively, they are harmonic in their domains. Moreover, equation (1.2) implies the transmission condition

$$
\alpha \partial_{\nu} u_{\alpha}(x)=\beta \partial_{\nu} u_{\beta}(x) \quad \text { for all } x \in \partial E \cap \Omega,
$$


where $\partial_{v}$ denotes the derivative of $u$ in the direction of the exterior normal to $\partial E$. Note that if $(E, u)$ is a smooth critical point of the functional (1.1) the following Euler-Lagrange equation holds

$$
\gamma H_{\partial E}+\beta\left|D u_{\beta}\right|^{2}-\alpha\left|D u_{\alpha}\right|^{2}=\lambda \quad \text { on } \partial E \cap \Omega,
$$

where $H_{\partial E}$ stands for the mean curvature of $\partial E$ and $\lambda$ is either zero or a Lagrange multiplier (in case of a volume constraint).

In the physical literature critical points of the functional (1.1), i.e, solutions of equations (1.2) and (1.4) are studied to model the shape of liquid drops exposed to an electric or a magnetic field. In the model the set $E$ represents a liquid drop with dielectric permittivity $\beta$, surrounded by a fluid with smaller permittivity $\alpha$, and $u$ stands for the electrostatic potential induced by an applied electric field. At the interface, which is assumed to be in static equilibrium, the normal component of the electric displacement field $\sigma_{E} D u$ has to be continuous. This implies that $u$ has to satisfy (1.3) or equivalently that it has to be a solution of equation (1.2). On the other hand, on the interface the electric stress and the surface tension have to be balanced, which leads to (1.4).

The occurrence of conical tips at an interface exposed to an electric field has been observed by several authors (see e.g. [21]). Moreover, theoretical investigations ( [14], [18], [19], [20]) suggest that conical critical points, the so called Taylor cones, may only occur if the ratio $\frac{\beta}{\alpha}$ is sufficiently large and if the opening angle is neither too small nor too close to $\pi / 2$, i.e., it belongs to a certain range which is independent of the penalization factor $\gamma$.

In order to state our first result we denote by $E_{\theta}$ the right circular cone with opening angle $\theta \in(0, \pi / 2)$ and vertex at the origin, i.e.,

$$
E_{\theta}=\left\{\left(x^{\prime}, x_{n}\right) \in \mathbb{R}^{n-1} \times \mathbb{R}: x_{n}>\frac{1}{\tan \theta}\left|x^{\prime}\right|\right\} .
$$

THEOREM 1.1 Let $n \geqslant 3$. There exist two positive constants $\delta_{0}=\delta_{0}\left(n, \frac{\beta}{\alpha}\right)>0$ and $\lambda_{0}=\lambda_{0}(n)>$ 1 such that, if $E_{\theta}$ is a right circular cone satisfying (1.4) then $\frac{\beta}{\alpha} \geqslant \lambda_{0}$ and

$$
\delta_{0} \leqslant \theta \leqslant \frac{\pi}{2}-\delta_{0}
$$

As far as we know, this result is the first rigorous proof of the fact that Taylor cones may occur only for certain angles, and provided that the ratio $\frac{\beta}{\alpha}$ is sufficiently large. We remark that we are able to give explicit estimates of the constants $\delta_{0}$ and $\lambda_{0}$. In particular, $\delta_{0}$ and $\lambda_{0}$ are independent of the penalization factor $\gamma$, which is in accordance with the observations and theoretical results reported in the physical literature.

The starting point in the proof of Theorem 1.1 is a rather simple decay estimate for the gradient of a minimizer of the Dirichlet energy (see Proposition 2.4). Roughly speaking, we prove that if $u_{E}$ is a solution of (1.2) and $x_{0}$ is a point in $\Omega$, where either the density of $E$ is close to 0 or 1 , or the set $E$ is asymptotically close to a hyperplane, then for sufficiently small $\rho$ we have

$$
\int_{B_{\rho}\left(x_{0}\right)}\left|D u_{E}\right|^{2} d x \leqslant C \rho^{n-\delta}
$$

for any $\delta>0$. As a consequence of this estimate one has that if the opening angle of the Taylor cone is not in the above range then the Dirichlet energy around the vertex decays faster than the perimeter thus leading to a contradiction to the criticality condition (1.4). 
In the mathematical literature people have also considered both the problem $(P)$ of minimizing (1.1) under the boundary condition $u=u_{0}$ on $\partial \Omega$ and the constrained problem

$$
\min \left\{\mathcal{F}(E, v): v=u_{0} \text { on } \partial \Omega,|E|=d\right\}
$$

for some given $d<|\Omega|$. The partial regularity of minimizers of the unconstrained problem $(P)$ was proved by Fan-Hua Lin in [15] (see also [1], [16]). In the special case $n=2$ the result of Lin has been improved by Larsen in [12], [13]. However, the full regularity of the free interface $\partial E$ in two dimensions still remains open.

In the second part of the paper we revisit the proof of the partial regularity of minimizers.

THEOREM 1.2 If $(E, u)$ is a minimizer of either problem $(P)$ or problem $\left(P_{c}\right)$, then

(a) there exists a relatively open set $\Gamma \subset \partial E$ such that $\Gamma$ is a $C^{1, \sigma}$ hypersurface for all $0<\sigma<$ $1 / 2$,

(b) there exists $\varepsilon>0$, depending only on $\frac{\beta}{\alpha}$ and $n$, such that $\mathcal{H}^{n-1-\varepsilon}((\partial E \backslash \Gamma) \cap \Omega)=0$.

The above statement slightly generalizes the regularity result proved in [15], where only the unconstrained problem $(P)$ was considered. The value of $\varepsilon$ is greater than or equal to $p-1$ where $2 p$ is the higher integrability exponent of $D u_{E}$ which is obtained by a standard application of Gehring's lemma (see Lemma 2.2). In particular, it is independent of the penalization factor $\gamma$. We note also that, once the $C^{1, \sigma}$ regularity of $\partial^{*} E \cap \Omega$ is obtained, then using [15, Lemma 5.3] and a standard bootstrap argument one obtains that $\partial^{*} E \cap \Omega$ is in fact $C^{\infty}$.

As in [15] and in the proof of the regularity of minimizers of the Mumford-Shah functional [3] our proof of Theorem 1.2 is based on an interplay between the perimeter and the Dirichlet integral. Differently from [15] we do not use the heavy machinery of currents and do not derive the monotonicity formula. Instead, our starting point is the same decay estimate (1.5) for the Dirichlet energy used for the study of Taylor cones. This estimate implies that if in a ball $B_{r}\left(x_{0}\right)$ the perimeter of $E$ is sufficiently small then the total energy in a smaller ball $B_{\tau r}\left(x_{0}\right)$

$$
P\left(E, B_{\tau r}\left(x_{0}\right)\right)+\int_{B_{\tau r}\left(x_{0}\right)}\left|D u_{E}\right|^{2} d x
$$

is much smaller than the total energy in $B_{r}\left(x_{0}\right)$ (Lemma 4.2). In turn this fact leads to a density lower bound for the perimeter.

Another consequence of the estimate (1.5) is that whenever the excess

$$
\varepsilon\left(x_{0}, r\right)=\inf _{\nu \in S^{n-1}} \frac{1}{r^{n-1}} \int_{\partial E \cap B_{r}\left(x_{0}\right)}\left|v_{E}(x)-v\right|^{2} d \mathcal{H}^{n-1} \rightarrow 0 \quad \text { as } r \rightarrow 0,
$$

the Dirichlet integral in $B_{r}\left(x_{0}\right)$ decays as in (1.5). As in the Mumford-Shah case this is one of the two key estimates needed for the regularity proof (see Step 1 of the proof of the Theorem 1.2 at the end of Section 4). Finally the excess decay is proven with a more or less standard argument similar to the one used for the $\Lambda$-minimizers of the perimeter.

After submitting this paper we learned that meanwhile De Philippis and Figalli gave in [6] a different proof of the estimate of the dimension of the singular set stated in Theorem 1.2 (b).

\section{Regularity of elastic minima}

In this section we study the regularity of the elastic minimum associated to a set $E$, i.e., solution of (1.2). In the main result of the section, Proposition 2.4, we prove that, if the density of $E$ is close to 
0 or 1 or the set $E$ is asymptotically close to a hyperplane, then the elastic energy $\int_{B_{\rho}\left(x_{0}\right)}|D u|^{2} d x$ decays faster that $\rho^{n-1}$. We prove Proposition 2.4 with a direct argument and therefore we are able to provide explicit bounds for the relevant constants.

We begin by deriving the Caccioppoli inequality for solutions of (1.2). Even though the argument is standard, we give the proof in order to keep track of the constants. We denote the cube, centred at $x_{0}$ and with side length $2 r$, by $Q_{r}\left(x_{0}\right)$. In the case $x_{0}=0$ we simply write $Q_{r}$. We recall the Sobolev-Poincaré inequality, i.e., for every function $u \in W^{1, p}\left(Q_{r}\right), 1 \leqslant p<n$, it holds

$$
\left\|u-u_{r}\right\|_{L^{p^{*}}\left(Q_{r}\right)} \leqslant c(n, p)\|D u\|_{L^{p}\left(Q_{r}\right)}
$$

where $u_{r}=f_{Q_{r}} u d x$ and $p^{*}=\frac{p n}{n-p}$.

LEMMA 2.1 Let $u \in W^{1,2}(\Omega)$ be a solution of (1.2). Then for every cube $Q_{2 r}\left(x_{0}\right) \subset \subset \Omega$ it holds

$$
f_{Q_{r}\left(x_{0}\right)}|D u|^{2} d x \leqslant C\left(f_{Q_{2 r}\left(x_{0}\right)}|D u|^{2 m} d x\right)^{\frac{1}{m}},
$$

where $m=\frac{n}{n+2}, C=C_{S, n}^{2} 2^{n+8} \frac{\beta}{\alpha}$, and $C_{S, n}$ is the constant in the Sobolev-Poincaré inequality (2.1) with $p=\frac{2 n}{n+2}$.

Proof. Without loss of generality we may assume that $x_{0}=0$. Let $\zeta \in C_{0}^{\infty}\left(Q_{2 r}\right)$ be a cut-off function such that $\zeta \equiv 1$ in $Q_{r}$ and $|D \zeta| \leqslant \frac{2}{r}$. We choose a test function $\varphi=\left(u-u_{2 r}\right) \zeta^{2}$ in (1.2), where $u_{2 r}=f_{Q_{2 r}} u d x$ and apply Young's inequality to obtain

$$
\int_{Q_{r}}|D u|^{2} d x \leqslant \int_{Q_{2 r}}|D u|^{2} \zeta^{2} d x \leqslant \frac{4 \beta}{\alpha} \int_{Q_{2 r}}\left|u-u_{2 r}\right|^{2}|D \zeta|^{2} d x \leqslant \frac{16 \beta}{r^{2} \alpha} \int_{Q_{2 r}}\left|u-u_{2 r}\right|^{2} d x .
$$

We use the Sobolev-Poincaré inequality (2.1) to deduce

$$
\int_{Q_{2 r}}\left|u-u_{2 r}\right|^{2} d x \leqslant C_{S, n}^{2}\left(\int_{Q_{2 r}}|D u|^{2 m} d x\right)^{\frac{1}{m}} .
$$

The result then follows from the two inequalities above.

We apply Gehring's Lemma to obtain higher integrability for the gradient of $u$.

LEMMA 2.2 Let $u \in W^{1,2}(\Omega)$ solve (1.2). There exists $p>1$ such that for any ball $B_{2 r}\left(x_{0}\right) \subset \subset \Omega$ it holds

$$
f_{B_{r}\left(x_{0}\right)}|D u|^{2 p} d x \leqslant C\left(f_{B_{2 r}\left(x_{0}\right)}|D u|^{2} d x\right)^{p} .
$$

The constants can be estimated explicitly as

$$
p=\frac{2 C_{1}-m}{2 C_{1}-1} \quad \text { for } C_{1}=C_{S, n}^{2} 2^{11} \cdot 80^{n} \frac{\beta}{\alpha} \quad \text { and } C=2^{2 n+1} 5^{n p} n^{n p / 2} \omega_{n}^{p-1},
$$

where $\omega_{n}$ is the volume of the unit ball and $m=\frac{n}{n+2}$.

The above result is well known but it is usually stated without estimates of the constants. In the Appendix we will go through the proof of Lemma 2.2 from [9] and evaluate every constant explicitly.

In the next lemma we prove a monotonicity formula for the elastic minimum in the case when $E$ is a half-space. 
LEMmA 2.3 Let $E=\left\{x \in \mathbb{R}^{n} \mid\langle x-\bar{x}, e\rangle<0\right\} \cap \Omega$ for some unit vector $e$ and a point $\bar{x}$, and suppose $u$ is a solution of (1.2). Let $x_{0} \in \partial E \cap \Omega$ and $r>0$ be such that $B_{r}\left(x_{0}\right) \subset \Omega$. Then

$$
\rho \mapsto f_{B_{\rho}\left(x_{0}\right)} \sigma_{E}(x)|D u|^{2} d x
$$

is increasing in $(0, r)$.

Proof. Without loss of generality we may assume that $E=\left\{x \in \mathbb{R}^{n} \mid x_{n}<0\right\} \cap \Omega$ and $x_{0}=0$. Let us fix a radius $r$ such that $B_{r} \subset \Omega$. From standard elliptic regularity theory we know that $u$ is smooth in the upper and in the lower part of the ball $B_{r}$ with respect to the hyperplane $\partial E=\left\{x_{n}=0\right\}$. To be more precise, let us denote $\bar{B}_{r}^{+}=\overline{B_{r} \backslash E}$ and $\bar{B}_{r}^{-}=\overline{B_{r} \cap E}$. Then $u_{\alpha} \in C^{\infty}\left(\bar{B}_{r}^{+}\right)$and $u_{\beta} \in C^{\infty}\left(\bar{B}_{r}^{-}\right)$and they are harmonic in the interior of $\bar{B}_{r}^{+}$and $\bar{B}_{r}^{-}$, where $u_{\alpha}$ and $u_{\beta}$ are the restrictions of $u$ to $\Omega \backslash E$ and $E$.

The goal is to show that the function $\varphi:(0, r) \rightarrow \mathbb{R}$

$$
\varphi(\rho):=f_{\partial B_{\rho}} \sigma_{E}(x)|D u(x)|^{2} d \mathcal{H}^{n-1}(x)=f_{\partial B_{1}} \sigma_{E}(\rho y)|D u(\rho y)|^{2} d \mathcal{H}^{n-1}(y)
$$

is increasing. Notice that $\sigma_{E}(\rho y)=\sigma_{E}(y)$ since $E$ is a half-space. Denote $v=|D u|^{2}, v_{\alpha}=$ $\left|D u_{\alpha}\right|^{2}$ and $v_{\beta}=\left|D u_{\beta}\right|^{2}$. Since $v_{\alpha}$ and $v_{\beta}$ are subharmonic in the interior of $B_{\rho}^{+}$and $B_{\rho}^{-}$we deduce by the divergence theorem that

$$
\begin{aligned}
\varphi^{\prime}(\rho)= & \int_{\partial B_{\rho}} \sigma_{E}(x)\left\langle D v(x), \frac{x}{\rho}\right\rangle d \mathcal{H}^{n-1}(x) \\
=\frac{1}{\mathcal{H}^{n-1}\left(\partial B_{\rho}\right)}\left(\alpha \int_{\partial B_{\rho}^{+}} \partial_{\nu} v_{\alpha} d \mathcal{H}^{n-1}\right. & +\beta \int_{\partial B_{\rho}^{-}} \partial_{\nu} v_{\beta} d \mathcal{H}^{n-1} \\
& \left.\quad+\int_{\partial E \cap B_{\rho}} \alpha \partial_{x_{n}} v_{\alpha}-\beta \partial_{x_{n}} v_{\beta} d \mathcal{H}^{n-1}\right) \\
= & \frac{1}{\mathcal{H}^{n-1}\left(\partial B_{\rho}\right)}\left(\alpha \int_{B_{\rho}^{+}} \Delta v_{\alpha} d \mathcal{H}^{n-1}+\beta \int_{B_{\rho}^{-}} \Delta v_{\beta} d \mathcal{H}^{n-1}\right. \\
& \left.\quad+\int_{\partial E \cap B_{\rho}} \alpha \partial_{x_{n}} v_{\alpha}-\beta \partial_{x_{n}} v_{\beta} d \mathcal{H}^{n-1}\right) \\
\geqslant & \frac{1}{\mathcal{H}^{n-1}\left(\partial B_{\rho}\right)} \int_{\partial E \cap B_{\rho}} \alpha \partial_{x_{n}} v_{\alpha}-\beta \partial_{x_{n}} v_{\beta} d \mathcal{H}^{n-1} .
\end{aligned}
$$

We will show that $\beta \partial_{x_{n}} v_{\beta}=\alpha \partial_{x_{n}} v_{\alpha}$ on $\partial E$ from which the claim follows.

Since $u_{\alpha}=u_{\beta}$ on $\partial E$ we have

$$
\partial_{x_{i}} u_{\alpha}=\partial_{x_{i}} u_{\beta} \quad \text { and } \quad \partial_{x_{i} x_{i}} u_{\alpha}=\partial_{x_{i} x_{i}} u_{\beta} \quad \text { for } i=1,2, \ldots, n-1 .
$$

The transmission condition (1.3) reads as

$$
\alpha \partial_{x_{n}} u_{\alpha}=\beta \partial_{x_{n}} u_{\beta} \quad \text { on } \partial E .
$$

Differentiating (2.3) with respect to $x_{i}$, for $i=1,2, \ldots, n-1$, yields

$$
\alpha \partial_{x_{i} x_{n}} u_{\alpha}=\beta \partial_{x_{i} x_{n}} u_{\beta} \quad \text { on } \partial E .
$$


On the other hand, since $u_{\alpha}$ and $u_{\beta}$ are harmonic, we have by (2.2) that

$$
\partial_{x_{n} x_{n}} u_{\alpha}=-\sum_{i=1}^{n-1} \partial_{x_{i} x_{i}} u_{\alpha}=-\sum_{i=1}^{n-1} \partial_{x_{i} x_{i}} u_{\beta}=\partial_{x_{n} x_{n}} u_{\beta} \quad \text { on } \partial E .
$$

Therefore on $\partial E$ it holds

$$
\alpha \partial_{x_{n}} v_{\alpha}=2 \sum_{i=1}^{n} \alpha \partial_{x_{i}} u_{\alpha} \partial_{x_{i} x_{n}} u_{\alpha}=2 \sum_{i=1}^{n} \beta \partial_{x_{i}} u_{\beta} \partial_{x_{i} x_{n}} u_{\beta}=\beta \partial_{x_{n}} v_{\beta}
$$

which implies $\varphi^{\prime}(\rho) \geqslant 0$.

The main result of this section is the following decay estimate for elastic minimum.

Proposition 2.4 Let $u \in W^{1,2}(\Omega)$ be a solution of (1.2). For all $\tau \in(0,1)$ there exists $\varepsilon_{0}=$ $\varepsilon_{0}(\tau)>0$ such that if $B_{r}\left(x_{0}\right) \subset \subset \Omega$ and one of the following conditions hold

(i) $\frac{\left|E \cap B_{r}\left(x_{0}\right)\right|}{\left|B_{r}\right|}<\varepsilon_{0}$,

(ii) $\frac{\left|B_{r}\left(x_{0}\right) \backslash E\right|}{\left|B_{r}\right|}<\varepsilon_{0}$,

(iii) there exists a half-space $H$ such that $\frac{\left|(E \Delta H) \cap B_{r}\left(x_{0}\right)\right|}{\left|B_{r}\right|}<\varepsilon_{0}$, then

$$
\int_{B_{\tau r}\left(x_{0}\right)}|D u|^{2} d x \leqslant C_{0} \tau^{n} \int_{B_{r}\left(x_{0}\right)}|D u|^{2} d x
$$

for some constant $C_{0}$ depending only on $\frac{\beta}{\alpha}$ and $n$.

Proof. Without loss of generality we may assume that $\tau<1 / 2$. We first treat the cases (i) and (ii). We fix a ball $B_{r}\left(x_{0}\right) \subset \subset \Omega$ and assume without loss of generality that $x_{0}=0$. Choose $v$ to be the harmonic function in $B_{r / 2}$ with the boundary condition $v=u$ on $\partial B_{r / 2}$. We choose the test function $\varphi=v-u \in W_{0}^{1,2}\left(B_{r / 2}\right)$ in the equations

$$
\int_{B_{r / 2}} D v \cdot D \varphi d x=0
$$

and

$$
\alpha \int_{B_{r / 2} \backslash E} D u \cdot D \varphi d x+\beta \int_{B_{r / 2} \cap E} D u \cdot D \varphi d x=0 .
$$

We write the latter equation as

$$
\int_{B_{r / 2}} D u \cdot(D v-D u) d x=-\frac{\beta-\alpha}{\alpha} \int_{B_{r / 2} \cap E} D u \cdot(D v-D u) d x .
$$

We substract from this $\int_{B_{r / 2}} D v \cdot(D v-D u) d x=0$ and use Hölder's inequality to deduce

$$
\int_{B_{r / 2}}|D v-D u|^{2} d x \leqslant \frac{(\beta-\alpha)^{2}}{\alpha^{2}} \int_{B_{r / 2} \cap E}|D u|^{2} d x .
$$


By the higher integrability stated in Lemma 2.2 we have

$$
\begin{aligned}
\int_{B_{\tau r}}|D v-D u|^{2} d x & \leqslant \frac{(\beta-\alpha)^{2}}{\alpha^{2}}\left|E \cap B_{r}\right|^{1-1 / p}\left|B_{r}\right|^{1 / p}\left(f_{B_{r / 2}}|D u|^{2 p}\right)^{1 / p} \\
& \leqslant C^{1 / p} \frac{(\beta-\alpha)^{2}}{\alpha^{2}}\left(\frac{\left|E \cap B_{r}\right|}{\left|B_{r}\right|}\right)^{1-1 / p} \int_{B_{r}}|D u|^{2} d x
\end{aligned}
$$

where $C$ and $p>1$ are from Lemma 2.2. Similarly we deduce

$$
\int_{B_{\tau r}}|D v-D u|^{2} d x \leqslant C^{1 / p} \frac{(\beta-\alpha)^{2}}{\beta^{2}}\left(\frac{\left|B_{r} \backslash E\right|}{\left|B_{r}\right|}\right)^{1-1 / p} \int_{B_{r}}|D u|^{2} d x .
$$

On the other hand, since $v$ is harmonic, we have

$$
\int_{B_{\tau r}}|D v|^{2} d x \leqslant 2^{n} \tau^{n} \int_{B_{r / 2}}|D v|^{2} d x \leqslant 2^{n} \tau^{n} \int_{B_{r / 2}}|D u|^{2} d x .
$$

Hence, we may estimate

$$
\begin{aligned}
\int_{B_{\tau r}}|D u|^{2} d x & \leqslant 2 \int_{B_{\tau r}}|D v-D u|^{2} d x+2 \int_{B_{\tau r}}|D v|^{2} d x \\
& \leqslant 2 \int_{B_{\tau r}}|D v-D u|^{2} d x+2^{n+1} \tau^{n} \int_{B_{r}}|D u|^{2} d x .
\end{aligned}
$$

If $\varepsilon_{0}$ is such that $\varepsilon_{0}^{1-\frac{1}{p}}=\tau^{n}$ then (2.5), (2.6) and (2.7) yield

$$
\int_{B_{\tau r}}|D u|^{2} d x \leqslant C \tau^{n} \int_{B_{r}}|D u|^{2} d x
$$

for a constant $C$ depending only on $\beta / \alpha$ and $n$.

We are left with the case (iii). Let $H$ be the half-space from the assumption. We choose $v$ which minimizes the energy $\int_{B_{r / 2}} \sigma_{H}(x)|D v|^{2} d x$ with the boundary condition $v=u$ on $\partial B_{r / 2}$. Hence

$$
\beta \int_{B_{r / 2} \cap H} D v \cdot D \varphi d x+\alpha \int_{B_{r / 2} \backslash H} D v \cdot D \varphi d x=0
$$

for every $\varphi \in W_{0}^{1,2}\left(B_{r / 2}\right)$. Lemma 2.3 yields

$$
\int_{B_{\tau r}}|D v|^{2} d x \leqslant \frac{\beta}{\alpha} 2^{n} \tau^{n} \int_{B_{r / 2}}|D v|^{2} d x .
$$

Moreover, from the minimality of $v$ it follows

$$
\int_{B_{r / 2}}|D v|^{2} d x \leqslant \frac{\beta}{\alpha} \int_{B_{r / 2}}|D u|^{2} d x .
$$


Hence, we have

$$
\int_{B_{\tau r}}|D u|^{2} d x \leqslant 2 \int_{B_{\tau r}}|D v-D u|^{2} d x+2^{n+1}\left(\frac{\beta}{\alpha}\right)^{2} \tau^{n} \int_{B_{r}}|D u|^{2} d x .
$$

Let us now rewrite the equation (2.4) satisfied by $u$ as

$$
\begin{aligned}
\beta \int_{B_{r / 2} \cap H} D u \cdot D \varphi d x+\alpha \int_{B_{r / 2} \backslash H} D u \cdot D \varphi d x \\
=(\beta-\alpha) \int_{B_{r / 2} \cap(H \backslash E)} D u \cdot D \varphi d x-(\beta-\alpha) \int_{B_{r / 2} \cap(E \backslash H)} D u \cdot D \varphi d x .
\end{aligned}
$$

Then, subtracting (2.8) from this equation and choosing $\varphi=u-v$ we get at once

$$
\int_{B_{\tau r}}|D u-D v|^{2} d x \leqslant\left(\frac{\beta}{\alpha}-1\right)^{2} \int_{B_{r / 2} \cap(E \Delta H)}|D u|^{2} d x
$$

and from Lemma 2.2 we deduce

$$
\int_{B_{\tau r}}|D u-D v|^{2} d x \leqslant C^{1 / p}\left(\frac{\beta}{\alpha}-1\right)^{2}\left(\frac{\left|(E \Delta H) \cap B_{r}\right|}{\left|B_{r}\right|}\right)^{1-1 / p} \int_{B_{r}}|D u|^{2} d x .
$$

The conclusion then follows as in the previous cases.

\section{Taylor cones}

In this section we study critical configurations $(E, u)$, i.e., configurations which satisfy (1.2) and (1.4). In particular, we are interested in those which are circular cones satisfying (1.4) outside the vertex. It was shown in [14] and [18] that there exist circular cones in $\mathbb{R}^{3}$

$$
E_{\theta_{0}}=\left\{x \in \mathbb{R}^{3} \mid x_{3}>\frac{1}{\tan \theta_{0}} \sqrt{x_{1}^{2}+x_{2}^{2}}\right\}
$$

for $\theta_{0} \in(0, \pi / 2)$, which are critical. Indeed, one may find an associated elastic minimum given in spherical coordinates by

$$
u(\rho, \theta)=\sqrt{\rho} \cdot f(\theta)
$$

where $\rho=\sqrt{x_{1}^{2}+x_{2}^{2}+x_{3}^{2}}$ and $\theta$ is the angle formed by the vector $x \in \mathbb{R}^{3}$ with the positive $x_{3}$ semi-axis. Denote by $P_{\frac{1}{2}}$ the Legendre function of the first kind of order $1 / 2$ which solves the equation

$$
P^{\prime \prime}(t)\left(1-t^{2}\right)-2 t P^{\prime}(t)+\frac{3}{4} P(t)=0, \quad t \in(-1,1) .
$$

Then the function $f$ is given by

$$
f(\theta)= \begin{cases}P_{\frac{1}{2}}\left(-\cos \theta_{0}\right) P_{\frac{1}{2}}(\cos \theta), & \theta \in\left[0, \theta_{0}\right] \\ P_{\frac{1}{2}}\left(\cos \theta_{0}\right) P_{\frac{1}{2}}(-\cos \theta), & \theta \in\left[\theta_{0}, \pi\right]\end{cases}
$$


The transmission condition (1.3) then reads as

$$
\beta P_{\frac{1}{2}}\left(-\cos \theta_{0}\right) P_{\frac{1}{2}}^{\prime}\left(\cos \theta_{0}\right)+\alpha P_{\frac{1}{2}}\left(\cos \theta_{0}\right) P_{\frac{1}{2}}^{\prime}\left(-\cos \theta_{0}\right)=0 .
$$

It can be proved that there exists a critical threshold $\lambda_{1} \approx 17.59$ such that this equation has no solutions if $\frac{\beta}{\alpha}<\lambda_{1}$ and it has two solutions in $(0, \pi / 2)$ if $\frac{\beta}{\alpha}>\lambda_{1}$.

In [19] estimates of the angles corresponding to critical cones are given by a different approach. Although the known results give sharp estimates for the angles which allow existence of critical spherical cones, they do not give any rigorous answer whether there exists a range of angles where no critical spherical cones appear.

We apply the regularity from Section 2 to prove that only cones with certain angles are possible. This estimate is independent of $\gamma$, which reflects the fact that the perimeter has only a regularizing effect. This result rigorously answers to the question connected to Taylor Cones, of why cones of certain angles do not appear. The result also generalizes to convex cones $E$ whose base is uniformly convex and $C^{2}$-regular. Since the result is purely local, with no loss of generality we set $\Omega=\mathbb{R}^{n}$.

We begin by revisiting the decay estimate proved in Proposition 2.4 with an explicit choice of the constants.

Proposition 3.1 Let $u \in W_{l o c}^{1,2}\left(\mathbb{R}^{n}\right)$ be a solution of (1.2). There exist $\delta_{1}, \sigma>0$ and $\vartheta \in(0,1)$, depending only on the dimension $n$ and the ratio $\frac{\beta}{\alpha}$, such that if one of the following conditions hold

(i) $\left|E \cap B_{1}\right|<\delta_{1}\left|B_{1}\right|$,

(ii) $\left|B_{1} \backslash E\right|<\delta_{1}\left|B_{1}\right|$,

(iii) there exists a half-space $H$ such that $\left|(E \Delta H) \cap B_{1}\right|<\delta_{1}\left|B_{1}\right|$, then we have

$$
\int_{B_{\vartheta}}|D u|^{2} d x \leqslant \vartheta^{n-1+\sigma} \int_{B_{1}}|D u|^{2} d x
$$

Proof. We first deal the case (i). We recall that from (2.5) and (2.7) it follows that if $\tau \in\left(0, \frac{1}{2}\right)$ then we have

$$
\int_{B_{\tau}}|D u|^{2} d x \leqslant 2\left(C^{1 / p} \frac{(\beta-\alpha)^{2}}{\alpha^{2}} \delta_{1}^{1-1 / p}+2^{n} \tau^{n}\right) \int_{B_{1}}|D u|^{2} d x
$$

where $C$ and $p$ are the constants from Lemma 2.2. Note that this inequality is trivially satisfied if $\tau \in\left(\frac{1}{2}, 1\right)$. Let us denote by $\chi$ the largest number such that the equation

$$
2 \chi+2^{n+1} \vartheta^{n}=\vartheta^{n-1}
$$

has a solution for some $\vartheta>0$. We may easily solve $\vartheta$

$$
\vartheta=\frac{n-1}{2^{n+1} n}
$$

Then if

$$
C^{1 / p} \frac{(\beta-\alpha)^{2}}{\alpha^{2}} \delta_{1}^{1-1 / p}<\chi
$$

we get

$$
\int_{B_{\vartheta}}|D u|^{2} d x \leqslant \vartheta^{n-1+\sigma} \int_{B_{1}}|D u|^{2} d x
$$


for some $\sigma>0$. Similarly in the case (ii) we have (3.2) provided that we choose $\chi$ and $\vartheta$ as in (3.1) and $\delta_{1}$ such that

$$
C^{1 / p} \frac{(\beta-\alpha)^{2}}{\beta^{2}} \delta_{1}^{1-1 / p}<\chi
$$

In the case (iii) we choose $\chi$ the largest number such that the equation

$$
2 \chi+2^{n+1}\left(\frac{\beta}{\alpha}\right)^{2} \vartheta^{n}=\vartheta^{n-1}
$$

has a solution $\vartheta>0$. We may again solve $\vartheta$

$$
\vartheta=\frac{n-1}{2^{n+1} n}\left(\frac{\alpha}{\beta}\right)^{2}
$$

Arguing as before, the estimate (3.2) follows from (2.9) and (2.10) if we choose $\delta_{1}$ such that

$$
C^{1 / p}\left(\frac{\beta}{\alpha}\right)^{2} \delta_{1}^{1-1 / p}<\chi
$$

By comparing the above three cases the claim obviously follows by choosing

$$
\delta_{1}^{1-1 / p}<\left(\frac{\alpha}{\beta}\right)^{2} C^{-1 / p} \chi
$$

where $\chi=\frac{1}{2} \vartheta^{n-1}-2^{n}\left(\frac{\beta}{\alpha}\right)^{2} \vartheta^{n}$ and $\vartheta$ is from (3.3).

We recall the following result (see the proof of [7, Theorem 3.1])

Proposition 3.2 Let $u \in W^{1,2}(\Omega)$ be a solution of (1.2). Let $x_{0} \in \Omega$ and $r>0$ such that $B_{r}\left(x_{0}\right) \subset \subset \Omega$. There exist $\lambda_{0}>1, c>0$ and $\sigma>0$, depending only on $n$, such that if

$$
\frac{\beta}{\alpha}<\lambda_{0}
$$

then for every $\rho<r$

$$
\int_{B_{\rho}\left(x_{0}\right)}|D u|^{2} d x \leqslant c\left(\frac{\rho}{r}\right)^{n-1+\sigma} \int_{B_{r}\left(x_{0}\right)}|D u|^{2} d x .
$$

We remark that we may estimate the number $\lambda_{0}$ (see $\left.[7,(20)-(21)]\right)$ by

$$
\lambda_{0}=\frac{n^{n}+n(n-1)^{n-1}-(n-1)^{n}}{n^{n}-n(n-1)^{n-1}+(n-1)^{n}} .
$$

Proof of Theorem 1.1. Let us recall the Euler-Lagrange equation for the critical set $E_{\theta}$

$$
\gamma H_{\partial E_{\theta}}+\beta\left|D u_{\beta}\right|^{2}-\alpha\left|D u_{\alpha}\right|^{2}=\lambda \quad \text { on } \partial E_{\theta} \backslash\{0\} .
$$

This can be rewritten as

$$
\gamma H_{\partial E_{\theta}}+\beta\left|\partial_{\nu} u_{\beta}\right|^{2}-\alpha\left|\partial_{\nu} u_{\alpha}\right|^{2}+(\beta-\alpha)\left|D_{\tau} u\right|^{2}=\lambda \quad \text { on } \partial E_{\theta} \backslash\{0\},
$$


where $D_{\tau} u$ is the tangential gradient of $u$ on $\partial E_{\theta} \backslash\{0\}$. From the transmission condition (1.3) we deduce

$$
\beta\left|\partial_{\nu} u_{\beta}\right|^{2}<\alpha\left|\partial_{\nu} u_{\alpha}\right|^{2} \quad \text { on } \partial E_{\theta} \backslash\{0\}
$$

Since $H_{\partial E_{\theta}}(x)=(n-2)(\tan \theta|x|)^{-1}$ for $x \in \partial E_{\theta} \backslash\{0\}$, we obtain from the Euler-Lagrange equation (3.7), from (3.8), and from the transmission condition (1.3) that

$$
\left|\partial_{\nu} u_{\beta}(x)\right| \geqslant \frac{c}{\sqrt{|x|}} \quad \text { on } \partial E_{\theta} \backslash\{0\}
$$

for some constant $c>0$. In particular, since the set $\partial E_{\theta} \backslash\{0\}$ is connected, this implies that $\partial_{\nu} u_{\beta}$ does not change sign on $\partial E_{\theta} \backslash\{0\}$, and we may thus assume it to be positive.

Let us fix $\rho>0$ and choose a cut-off function $\zeta \in C_{0}^{\infty}\left(B_{\rho}\right)$ such that $\zeta \equiv 1$ in $B_{\rho / 2}$ and $|D \zeta| \leqslant 4 / \rho$. Since $u_{\beta}$ is harmonic in $E_{\theta}$, we obtain from (3.9) and by integrating by parts that

$$
\int_{E_{\theta} \cap B_{\rho}}\left\langle D u_{\beta}, D \zeta\right\rangle d x=\int_{\partial E_{\theta} \cap B_{\rho}} \partial_{\nu} u_{\beta} \zeta d \mathcal{H}^{n-1} \geqslant c \int_{\partial E_{\theta} \cap B_{\rho / 2}}|x|^{-1 / 2} d \mathcal{H}^{n-1} \geqslant \tilde{c} \rho^{n-3 / 2} .
$$

On the other hand Hölder's inequality implies

$$
\begin{aligned}
\int_{E_{\theta} \cap B_{\rho}}\left\langle D u_{\beta}, D \zeta\right\rangle d x & \leqslant\left(\int_{E_{\theta} \cap B_{\rho}}|D \zeta|^{2} d x\right)^{1 / 2}\left(\int_{E_{\theta} \cap B_{\rho}}\left|D u_{\beta}\right|^{2} d x\right)^{1 / 2} \\
& \leqslant C \rho^{n / 2-1}\left(\int_{E_{\theta} \cap B_{\rho}}\left|D u_{\beta}\right|^{2} d x\right)^{1 / 2}
\end{aligned}
$$

Therefore

$$
\int_{E_{\theta} \cap B_{\rho}}\left|D u_{\beta}\right|^{2} d x \geqslant c_{0} \rho^{n-1}
$$

for some constant $c_{0}>0$. Proposition 3.2 implies that $\frac{\beta}{\alpha} \geqslant \lambda_{0}$. Moreover Proposition 3.1 (i) and (iii) with a standard iteration argument imply that $\delta_{1}\left|B_{1}\right| \leqslant\left|E_{\theta} \cap B_{1}\right|$ and $\delta_{1}\left|B_{1}\right| \leqslant \mid B_{1} \cap\left(\left\{x_{n}>\right.\right.$ $\left.0\} \backslash E_{\theta}\right) \mid$. Hence, the conclusion follows.

REMARK 3.3 The constant $\delta_{0}$ can be explicitly estimated in terms of the constant $\delta_{1}$ from Proposition 3.1, since the spherical sector has the volume

$$
\left|E_{\theta} \cap B_{1}\right|=\omega_{n-1}\left(\int_{0}^{\theta} \sin ^{n} t d t+\frac{\sin ^{n-1} \theta \cos \theta}{n}\right),
$$

where $\omega_{n-1}$ is the volume of the $(n-1)$-dimensional unit ball. The formula for $\delta_{1}$ is given by (3.5). The constant $\lambda_{0}$ is estimated in (3.6).

Note that in dimension 2 the Euler-Lagrange equation (1.4) reduces to

$$
\beta\left|D u_{\beta}\right|^{2}-\alpha\left|D u_{\alpha}\right|^{2}=\lambda \quad \text { on } \partial E_{\theta} \backslash\{0\}
$$

and it is not clear to us if this weaker information is enough to establish Theorem 1.1 also in this case. However, as we already mentioned earlier, for $n \geqslant 3$, the proof of that theorem can be easily generalized to more general cones. 
REMARK 3.4 Let $K \subset \mathbb{R}^{n-1}$ be an open, uniformly convex and $C^{2}$-regular set such that $0 \in K$. Theorem 1.1 can be generalized to conical sets of the form

$$
E=\left\{\lambda\left(x^{\prime}, 1\right) \in \mathbb{R}^{n} \mid x^{\prime} \in K, \lambda \geqslant 0\right\}
$$

More precisely, if $E$ satisfies (1.4) then $\frac{\beta}{\alpha} \geqslant \lambda_{0}$ and $\delta_{1}\left|B_{1}\right| \leqslant\left|E \cap B_{1}\right|$ and $\delta_{1}\left|B_{1}\right| \leqslant \mid B_{1} \cap\left(\left\{x_{n}>\right.\right.$ $0\} \backslash E) \mid$ where $\delta_{1}$ is as in Proposition 3.1. The proof is identical to the one given before as soon as one observes that there exists $c>0$ such that for all $x \in \partial E \backslash\{0\}, H_{\partial E}(x) \geqslant \frac{c}{|x|}$.

\section{Regularity of minimizers}

Throughout this section the dimension $n$ and the constants $\alpha, \beta$ and $\gamma$ will remain fixed. Thus we denote by $C$ a generic constant depending on these quantities and whose value may change from line to line. On the other hand special constants will be numbered and their dependence on other quantities will be explicitly mentioned. Before proceeding in the regularity proof we recall the following result which was proved in [7, Theorem 1].

THEOREM 4.1 Let $(E, u)$ be a minimizer of problem $\left(P_{c}\right)$. There exists a constant $\Lambda$ such that $(E, u)$ is also a minimizer of the penalized functional

$$
\mathcal{F}_{\Lambda}(F, v)=\gamma P(F, \Omega)+\int_{\Omega} \sigma_{F}(x)|D v|^{2} d x+\Lambda|| F|-| E||
$$

among all $(F, v)$ such that $v=u$ on $\partial \Omega$.

Motivated by the previous theorem we give the following definition. To this aim we denote for a set $E$ with finite perimeter in $\Omega$ and a function $u \in W^{1,2}(\Omega)$ by

$$
\mathcal{F}(E, u ; U)=\gamma P(E, U)+\int_{U} \sigma_{E}|D u|^{2} d x
$$

the energy of the pair $(E, u)$ in an open set $U \subset \subset \Omega$. We say that a pair $(E, u)$ is a $\Lambda$-minimizer of $\mathcal{F}$ in $\Omega$ if for every $B_{r}\left(x_{0}\right) \subset \Omega$ and every pair $(F, v)$, where $F$ is a set of finite perimeter with $F \Delta E \subset \subset B_{r}\left(x_{0}\right)$ and $v-u \in W_{0}^{1,2}\left(B_{r}\left(x_{0}\right)\right)$, we have

$$
\mathcal{F}\left(E, u ; B_{r}\left(x_{0}\right)\right) \leqslant \mathcal{F}\left(F, v ; B_{r}\left(x_{0}\right)\right)+\Lambda|F \Delta E| .
$$

Note that any minimizing pair $\left(E, u_{E}\right)$ of the constrained problem $\left(P_{c}\right)$ is a $\Lambda$-minimizer of $\mathcal{F}$ for some $\Lambda$. Since $\Lambda$ will be fixed from now on the dependence of the constants on $\Lambda$ will not be highlighted.

LEMMA 4.2 Let $(E, u)$ be a $\Lambda$-minimizer of $\mathcal{F}$ in $\Omega$. For every $\tau \in(0,1)$ there exists $\varepsilon_{1}=\varepsilon_{1}(\tau)>$ 0 such that if $B_{r}\left(x_{0}\right) \subset \Omega$ and $P\left(E ; B_{r}\left(x_{0}\right)\right)<\varepsilon_{1} r^{n-1}$ then

$$
\mathcal{F}\left(E, u ; B_{\tau r}\left(x_{0}\right)\right) \leqslant C_{1} \tau^{n}\left(\mathcal{F}\left(E, u ; B_{r}\left(x_{0}\right)\right)+r^{n}\right)
$$

for some constant $C_{1}$ independent of $\tau$ and $r$. 
Proof. If we replace $E$ by $\frac{E-x_{0}}{r}, u$ by $y \rightarrow r^{-1 / 2} u\left(x_{0}+r y\right)$ and $\Lambda$ by $\Lambda r$ we may assume that $r=1, x_{0}=0$ and $(E, u)$ is a $\Lambda r$-minimizer in $\frac{\Omega-x_{0}}{r}$. After this rescaling we are left to prove that for a given $\tau \in(0,1)$ there exists $\varepsilon_{1}=\varepsilon_{1}(\tau)>0$ such that if $P\left(E, B_{1}\right)<\varepsilon_{1}$ then

$$
\mathcal{F}\left(E, u ; B_{\tau}\right) \leqslant C_{1} \tau^{n}\left(\mathcal{F}\left(E, u ; B_{1}\right)+\Lambda r\right) .
$$

Without loss of generality we may assume that $\tau<1 / 2$.

If the perimeter of $E$ in $B_{1}$ is small then, by the relative isoperimetric inequality, either $\left|B_{1} \cap E\right|$ or $\left|B_{1} \backslash E\right|$ is small. Assume the latter is true. Then we have

$$
\left|B_{1} \backslash E\right| \leqslant c(n) P\left(E, B_{1}\right)^{\frac{n}{n-1}} .
$$

By Fubini's theorem and choosing as a representative of $E$ the set of points of density one, we have

$$
\left|B_{1} \backslash E\right| \geqslant \int_{\tau}^{2 \tau} \mathcal{H}^{n-1}\left(\partial B_{\rho} \backslash E\right) d \rho .
$$

Therefore we may choose $\rho \in(\tau, 2 \tau)$ such that

$$
\mathcal{H}^{n-1}\left(\partial B_{\rho} \backslash E\right) \leqslant \frac{c(n)}{\tau} P\left(E, B_{1}\right)^{\frac{n}{n-1}} \leqslant \frac{c(n) \varepsilon_{1}^{\frac{1}{n-1}}}{\tau} P\left(E, B_{1}\right) .
$$

Set $F=E \cup B_{\rho}$ and observe that by the choice of the representative of $E$ it holds

$$
P\left(F ; B_{1}\right) \leqslant P\left(E, B_{1} \backslash B_{\rho}\right)+\mathcal{H}^{n-1}\left(\partial B_{\rho} \backslash E\right) .
$$

Choosing $(F, u)$ as a competing pair and using the $\Lambda r$-minimality of $(E, u)$ we get

$$
\begin{aligned}
\gamma P\left(E, B_{1}\right)+\int_{B_{1}} \sigma_{E}|D u|^{2} d x & \leqslant \gamma P\left(F ; B_{1}\right)+\int_{B_{1}} \sigma_{F}|D u|^{2} d x+\Lambda r|F \backslash E| \\
& \leqslant \gamma\left(P\left(E, B_{1} \backslash B_{\rho}\right)+\mathcal{H}^{n-1}\left(\partial B_{\rho} \backslash E\right)\right)+\int_{B_{1}} \sigma_{F}|D u|^{2} d x+\Lambda r\left|B_{\rho}\right| .
\end{aligned}
$$

Hence, choosing $\varepsilon_{1}$ such that $c(n) \varepsilon_{1}^{\frac{1}{n-1}} \leqslant \tau^{n+1}$ and recalling that $\rho \in(\tau, 2 \tau)$ we get from (4.1) that

$$
\gamma P\left(E, B_{\tau}\right)+\int_{B_{\tau}} \sigma_{E}|D u|^{2} d x \leqslant \gamma \tau^{n} P\left(E, B_{1}\right)+\beta \int_{B_{2 \tau}}|D u|^{2} d x+\Lambda r\left|B_{2 \tau}\right| .
$$

If we choose $\varepsilon_{1}$ such that $c(n) \varepsilon_{1}^{\frac{n}{n-1}} \leqslant \varepsilon_{0}(2 \tau)\left|B_{1}\right|$, where $\varepsilon_{0}$ is from Proposition 2.4, we get

$$
\int_{B_{2 \tau}}|D u|^{2} d x \leqslant 2^{n} C_{0} \tau^{n} \int_{B_{1}}|D u|^{2} d x
$$

and the result follows.

The next result is contained in [15, Theorem 2], where it is proven for local minimizers of $\mathcal{F}$. However, in the case of $\Lambda$-minimizers the same proof applies without changes once one observes that by comparing $(E, u)$ with $(F, u)$, where $F=E \backslash B_{r}\left(x_{0}\right)$ and $B_{r}\left(x_{0}\right) \subset \subset \Omega$, one gets

$$
\gamma P\left(E, B_{r}\left(x_{0}\right)\right)+(\beta-\alpha) \int_{E \cap B_{r}\left(x_{0}\right)}|D u|^{2} d x \leqslant \gamma \mathcal{H}^{n-1}\left(\partial B_{r} \cap E\right)+\Lambda\left|B_{r}\right| \leqslant C r^{n-1} .
$$


THEOREM 4.3 Let $(E, u)$ be a $\Lambda$-minimizer of $\mathcal{F}$ in $\Omega$. For every open set $U \subset \subset \Omega$ there exists a constant $C_{2}$, depending on $U$ and $\|D u\|_{L^{2}(\Omega)}$, such that for every $B_{r}\left(x_{0}\right) \subset U$ it holds

$$
\mathcal{F}\left(E, u ; B_{r}\left(x_{0}\right)\right) \leqslant C_{2} r^{n-1} .
$$

By combining the energy decay Lemma 4.2 with the energy upper bound given by Theorem 4.3 we obtain the following density lower bound by a standard iteration argument. From now on the topological boundary $\partial E$ must be understood by considering the correct representative of the set (see [17, Proposition 12.19]). In particular, for such representative of $E$ it holds $\overline{\partial^{*} E}=\partial E$, where $\partial^{*} E$ denotes the reduced boundary of $E$.

Proposition 4.4 Let $(E, u)$ be a $\Lambda$-minimizer of $\mathcal{f}$ and $U \subset \subset \Omega$. There exists a constant $c>0$, depending on $U$ and $\|D u\|_{L^{2}(\Omega)}$, such that for every $B_{r}\left(x_{0}\right) \subset U$ with $x_{0} \in \partial E$ we have

$$
P\left(E, B_{r}\left(x_{0}\right)\right) \geqslant c r^{n-1} .
$$

Moreover $\mathcal{H}^{n-1}\left(\left(\partial E \backslash \partial^{*} E\right) \cap \Omega\right)=0$.

Proof. The proof is as in [3, Theorem 7.21] and in fact, even simpler. Fix $x_{0} \in \partial^{*} E$. Without loss of generality we may assume that $x_{0}=0$. Fix $\tau \in(0,1)$ such that $2 C_{1} \tau^{\frac{1}{2}}<1$ and $\sigma \in(0,1)$ such that $2 C_{1} C_{2} \sigma<\varepsilon_{1}(\tau) \gamma$ and $r_{0}$ such that $r_{0}<\min \left\{\varepsilon_{1}(\tau) \gamma, C_{2}\right\}$, where $C_{1}, \varepsilon_{1}$ and $C_{2}$ are the constants from Lemma 4.2 and Theorem 4.3. Assume by contradiction that for some $B_{r} \subset U$ with $r<r_{0}$ we have $P\left(E, B_{r}\right)<\varepsilon_{1}(\sigma) r^{n-1}$. Then by induction we deduce that

$$
\mathcal{F}\left(E, u ; B_{\sigma \tau^{h} r}\right) \leqslant \varepsilon_{1}(\tau) \gamma \tau^{\frac{h}{2}}\left(\sigma \tau^{h} r\right)^{n-1}
$$

for all $h=0,1,2, \ldots$ Indeed, when $h=0$, using Lemma 4.2 and Theorem 4.3 and recalling our choice of $\sigma$ and $r_{0}$ we obtain

$$
\mathcal{F}\left(E, u ; B_{\sigma r}\right) \leqslant C_{1} \sigma^{n}\left(\mathfrak{F}\left(E, u ; B_{r}\right)+r^{n}\right) \leqslant 2 C_{1} C_{2} \sigma(\sigma r)^{n-1}<\varepsilon_{1}(\tau) \gamma(\sigma r)^{n-1} .
$$

If (4.2) holds for $h$ to deduce that it also holds for $h+1$ it is enough to apply Lemma 4.2 and to recall that $2 C_{1} \tau^{\frac{1}{2}}<1$ and that $r<r_{0}<\varepsilon_{1}(\tau) \gamma$. In particular, this implies

$$
\lim _{r \rightarrow 0} \frac{P\left(E, B_{r}\right)}{r^{n-1}}=0,
$$

which is a contradiction since $x_{0} \in \partial^{*} E$. This proves that for every $x_{0} \in \partial^{*} E$ and $r<r_{0}$ it holds $P\left(E, B_{r}\left(x_{0}\right)\right) \geqslant \varepsilon_{1}(\sigma) r^{n-1}$. The claim follows from the fact that $\overline{\partial^{*} E}=\partial E$. The fact that $\mathcal{H}^{n-1}\left(\left(\partial E \backslash \partial^{*} E\right) \cap \Omega\right)=0$ follows from the density lower bound and [3, (2.42)].

Let $(E, u)$ be a $\Lambda$-minimizer of $\mathcal{F}$. We introduce the excess of $E$ at the point $x \in \partial E$ at the scale $r>0$ in direction $v \in S^{n-1}$

$$
\mathcal{E}(x, r, v):=\frac{1}{2 r^{n-1}} \int_{\partial E \cap B_{r}(x)}\left|v_{E}(y)-v\right|^{2} d \mathcal{H}^{n-1}(y)
$$

and

$$
\varepsilon(x, r):=\min _{v \in S^{n-1}} \varepsilon(x, r, v)
$$


and the rescaled Dirichlet integral of $u$

$$
Ð(x, r):=\frac{1}{r^{n-1}} \int_{B_{r}(x)}|D u|^{2} d y \text {. }
$$

The density lower bound implies the following important properties of $\Lambda$-minimizers of $\mathfrak{F}$. The first one is the so called height bound lemma.

LemMA 4.5 (Height bound) Let $(E, u)$ be a $\Lambda$-minimizer of $\mathcal{F}$ in $B_{r}\left(x_{0}\right)$. There exist $C$ and $\varepsilon>0$, depending on $\|D u\|_{L^{2}\left(B_{r}\left(x_{0}\right)\right)}$, such that if $x_{0} \in \partial E$ and

$$
\varepsilon\left(x_{0}, r, v\right)<\varepsilon
$$

for some $v \in S^{n-1}$ then

$$
\sup _{y \in \partial E \cap B_{r / 2}\left(x_{0}\right)} \frac{\left|v \cdot\left(y-x_{0}\right)\right|}{r} \leqslant C \varepsilon\left(x_{0}, r, v\right)^{\frac{1}{2(n-1)}} .
$$

Proof. The proof of this result can be obtained arguing exactly as in the case of $\Lambda$-minimizers of the perimeter [17, Theorem 22.8]. Indeed, it is based only on the relative isoperimetric inequality, the density lower bound, i.e., Proposition 4.4 and the compactness result below.

The next result is proved as in the case of the $\Lambda$-minimizers of the perimeter with the obvious changes due to the presence of the Dirichlet integral.

LEMMA 4.6 (Compactness) Let $\left(E_{h}, u_{h}\right)$ be a sequence of $\Lambda_{h}$-minimizers of $\mathcal{F}$ in $\Omega$ such that $\Lambda_{h} \rightarrow \Lambda \in[0, \infty)$ and $\sup _{h} \mathcal{F}\left(E_{h}, u_{h} ; \Omega\right)<\infty$. Then there exist a subsequence, not relabeled, and a $\Lambda$-minimizer $(E, u)$ of $\mathcal{F}$ such that for every open set $U \subset \subset \Omega, E_{h} \rightarrow E$ in $L^{1}(U), P\left(E_{h}, U\right) \rightarrow$ $P(E, U), u_{h} \rightarrow u$ in $W^{1,2}(U)$ and moreover

(i) if $x_{h} \in \partial E_{h} \cap U$ and $x_{h} \rightarrow x \in U$, then $x \in \partial E \cap U$.

(ii) if $x \in \partial E \cap U$ there exists $x_{h} \in \partial E_{h} \cap U$ such that $x_{h} \rightarrow x$.

If in addition, $D u_{h} \rightarrow 0$ weakly in $L_{l o c}^{2}\left(\Omega, \mathbb{R}^{n}\right)$ and $\Lambda_{h} \rightarrow 0$, then $E$ is a local minimizer of the perimeter, i.e., for every $F$ such that $F \Delta E \subset \subset B_{r}\left(x_{0}\right) \subset \Omega$ it holds

$$
P\left(E, B_{r}\left(x_{0}\right)\right) \leqslant P\left(F, B_{r}\left(x_{0}\right)\right) \text {. }
$$

Proof. We start by proving the $\Lambda$-minimality of $(E, u)$. Let us fix $B_{r}\left(x_{0}\right) \subset \subset \Omega$ and assume that $x_{0}=0$. Without loss of generality we may assume that $E_{h} \rightarrow E$ in $L^{1}\left(B_{r}\right), u_{h} \rightarrow u$ weakly in $W^{1,2}\left(B_{r}\right)$ and strongly in $L^{2}\left(B_{r}\right)$. Let $(F, v)$ be a pair such that $F \Delta E \subset \subset B_{r}$ and $\operatorname{supp}(u-v) \subset \subset B_{r}$. By Fubini's theorem and passing to a subsequence if necessary we may choose $\rho<r$ such that $F \Delta E \subset \subset B_{\rho}, \operatorname{supp}(u-v) \subset \subset B_{\rho}$,

$$
\mathcal{H}^{n-1}\left(\partial^{*} F \cap \partial B_{\rho}\right)=\mathcal{H}^{n-1}\left(\partial^{*} E_{h} \cap \partial B_{\rho}\right)=0 \text { and } \lim _{h \rightarrow 0} \mathcal{H}^{n-1}\left(\partial B_{\rho} \cap E \Delta E_{h}\right)=0
$$

where it is understood that $E$ and $E_{h}$ stand for the set of points of density one of $E$ and $E_{h}$, respectively. Fix a cut-off function $\zeta \in C_{0}^{1}\left(B_{r}\right)$ such that $\zeta \equiv 1$ in $B_{\rho}$, and choose $F_{h}=(F \cap$ $\left.B_{\rho}\right) \cup\left(E_{h} \backslash B_{\rho}\right)$ and $v_{h}=\zeta v+(1-\zeta) u_{h}$. Then by the $\Lambda_{h}$-minimality of $\left(E_{h}, u_{h}\right)$, the choice of 
$\rho$, the strong convergence of $u_{h} \rightarrow u$ in $L^{2}$ and by convexity, we have

$$
\begin{aligned}
\gamma P\left(E_{h}, B_{r}\right)+\int_{B_{r}} \sigma_{E_{h}}\left|D u_{h}\right|^{2} d x \leqslant & \gamma\left(F_{h}, B_{r}\right)+\int_{B_{r}} \sigma_{F_{h}}\left|D v_{h}\right|^{2} d x+\Lambda_{h}\left|F_{h} \Delta E_{h}\right| \\
\leqslant & \gamma\left[P\left(F, B_{\rho}\right)+P\left(E_{h}, B_{r} \backslash B_{\rho}\right)\right]+\int_{B_{r}} \sigma_{F_{h}} \zeta|D v|^{2} d x \\
& +\int_{B_{r}} \sigma_{E_{h}}(1-\zeta)\left|D u_{h}\right|^{2} d x+\varepsilon_{h}+\Lambda_{h}\left|F_{h} \Delta E_{h}\right|
\end{aligned}
$$

for some $\varepsilon_{h} \rightarrow 0$. Thus by a simple lower semicontinuity argument we have

$$
\gamma P\left(E, B_{\rho}\right)+\int_{B_{r}} \sigma_{E} \zeta|D u|^{2} d x \leqslant \gamma P\left(F, B_{\rho}\right)+\int_{B_{r}} \sigma_{F} \zeta|D v|^{2} d x+\Lambda|F \Delta E| .
$$

Letting $\zeta \downarrow \chi_{B_{\rho}}$ in the previous inequality we conclude that

$$
\gamma P\left(E, B_{\rho}\right)+\int_{B_{\rho}} \sigma_{E}|D u|^{2} d x \leqslant \gamma P\left(F, B_{\rho}\right)+\int_{B_{\rho}} \sigma_{F}|D v|^{2} d x+\Lambda|F \Delta E|
$$

thus proving the $\Lambda$-minimality of $(E, u)$. Similarly, choosing $F=E$ and $v=u$ in (4.3) and arguing as before we get

$$
\limsup _{h \rightarrow \infty}\left(\gamma P\left(E_{h}, B_{\rho}\right)+\int_{B_{r}} \sigma_{E_{h}} \zeta\left|D u_{h}\right|^{2} d x\right) \leqslant \gamma P\left(E, B_{\rho}\right)+\int_{B_{r}} \sigma_{E} \zeta|D u|^{2} d x .
$$

Letting $\zeta \downarrow \chi_{B_{\rho}}$ we conclude that

$$
\lim _{h \rightarrow \infty} P\left(E_{h}, B_{\rho}\right)=P\left(E, B_{\rho}\right) \quad \text { and } \quad \lim _{h \rightarrow \infty} \int_{B_{\rho}} \sigma_{E_{h}}\left|D u_{h}\right|^{2} d x=\int_{B_{\rho}} \sigma_{E}|D u|^{2} d x .
$$

A standard argument then implies that $P\left(E_{h}, U\right) \rightarrow P(E, U), u_{h} \rightarrow u$ in $W^{1,2}(U)$ for every open set $U \subset \subset \Omega$.

Finally we note that if $D u=0$ we can choose $v=u$ in (4.4) thus proving that $E$ is a $\Lambda$ minimizer of the perimeter. The claim (i) and (ii) follow exactly as in [17, Theorem 21.14].

The next consequence of the density lower bound is the Lipschitz approximation. Its proof is based only on the height bound estimate and can be obtained by following word by word the proof given in [17, Theorem 23.7]. To that aim we use the notation $x=\left(x^{\prime}, x_{n}\right) \in \mathbb{R}^{n-1} \times \mathbb{R}$ for a generic point in $\mathbb{R}^{n}$ and with a slight abuse of notation we still denote by $D f$ the gradient of a function $f: \mathbb{R}^{n-1} \rightarrow \mathbb{R}$. If $x^{\prime} \in \mathbb{R}^{n-1}$, the $(n-1)$-dimensional ball with center $x^{\prime}$ and radius $r$ will be denoted by $B_{r}^{(n-1)}\left(x^{\prime}\right)$.

Proposition 4.7 (Lipschitz approximation) Let $(E, u)$ be a $\Lambda$-minimizer of $\mathcal{F}$ in $B_{r}$. There exist $C_{3}$ and $\varepsilon_{3}>0$, depending on $\|D u\|_{L^{2}\left(B_{r}\right)}$, such that if $0 \in \partial E$ and

$$
\mathcal{E}\left(0, r, e_{n}\right)<\varepsilon_{3}
$$

then there exists a Lipschitz function $f: \mathbb{R}^{n-1} \rightarrow \mathbb{R}$ such that

$$
\sup _{x^{\prime} \in \mathbb{R}^{n-1}} \frac{\left|f\left(x^{\prime}\right)\right|}{r} \leqslant C_{3} \varepsilon\left(0, r, e_{n}\right)^{\frac{1}{2(n-1)}}, \quad\|D f\|_{L^{\infty}} \leqslant 1
$$


and

$$
\frac{1}{r^{n-1}} \mathcal{H}^{n-1}\left(\partial E \Delta \Gamma_{f} \cap B_{r / 2}\right) \leqslant C_{3} \mathcal{E}\left(0, r, e_{n}\right)
$$

where $\Gamma_{f}$ is the graph of $f$. Moreover

$$
\frac{1}{r^{n-1}} \int_{B_{r / 2}^{n-1}}|D f|^{2} d x^{\prime} \leqslant C_{3} \varepsilon\left(0, r, e_{n}\right) .
$$

Finally we state the following reverse Poincaré inequality which plays the role of the classical Caccioppoli inequality in the elliptic regularity theory.

THEOREM 4.8 Let $(E, u)$ be a $\Lambda$-minimizer of $\mathcal{F}$ in $B_{r}\left(x_{0}\right)$. There exist two constants $C_{4}$ and $\varepsilon_{4}>0$ such that if $x_{0} \in \partial E$ and $\varepsilon\left(x_{0}, r, v\right)<\varepsilon_{4}$ then

$$
\mathcal{E}\left(x_{0}, r / 2, v\right) \leqslant C_{4}\left(\frac{1}{r^{n+1}} \int_{\partial E \cap B_{r}\left(x_{0}\right)}\left|v \cdot\left(x-x_{0}\right)-c\right|^{2} d \mathcal{H}^{n-1}+\Phi\left(x_{0}, r\right)+r\right)
$$

for every $c \in \mathbb{R}$.

Proof. The proof can be obtained exactly as in the case of $\Lambda$-minimizers of the perimeter (see [17, Theorem 24.1]) with the following minor changes.

For every $z \in \mathbb{R}^{n-1}, s>0$, we denote by $C_{s}(z)$ and $K_{s}(z)$ the cylinders

$$
C_{s}(z)=B_{s}^{n-1}(z) \times(-s, s), \quad K_{s}(z)=B_{s}^{n-1}(z) \times(-1,1) .
$$

The first step in the proof is to establish a weak form of the inequality (4.5). Indeed one can prove that if $(E, u)$ is a $\Lambda$-minimizer of $\mathcal{F}$ in $C_{4}=C_{4}(0)$ such that $\left|x_{n}\right| \leqslant 1 / 8$ for all $x \in \partial E \cap C_{2}$ and

$$
\left|\left\{x \in C_{2} \backslash E: x_{n}<-1 / 8\right\}\right|=\left|\left\{x \in C_{2} \cap E: x_{n}>1 / 8\right\}\right|=0
$$

and if for some $z \in \mathbb{R}^{n-1}$ and $s>0$ one has

$$
K_{2 s}(z) \subset C_{2}, \quad \mathcal{H}^{n-1}\left(\partial E \cap K_{2 s}(z)\right)=0,
$$

then for any $|c|<1 / 4$

$$
\begin{aligned}
P\left(E, K_{s}(z)\right)-\mathcal{H}^{n-1}\left(B_{s}^{n-1}\right) & \\
\leqslant C(n)\left(\left(P\left(E, K_{2 s}(z)\right)-\mathcal{H}^{n-1}\left(B_{2 s}^{n-1}\right)\right)\right. & \left.\int_{K_{2 s}(z)} \frac{\left|x_{n}-c\right|^{2}}{s^{2}} d \mathcal{H}^{n-1}\right)^{1 / 2} \\
& +C(n) \Lambda s^{n-1}+C(n) \int_{K_{2 s}(z)}|D u|^{2} d x .
\end{aligned}
$$

The proof of this inequality is exactly as in [17, Lemma 24.9] with the obvious choice of the comparison configuration equal to $\left(G_{k}, u\right)$ where $G_{k}$ is as in proof of [17, Lemma 24.9].

Let us now prove (4.5). To this aim, by the usual translation and rescaling argument, we may assume that $x_{0}=0, r=1$, that $(E, u)$ is $\Lambda r$-minimizer of $\mathcal{F}$ and that $v=e_{n}$. With the above 
inequality in hands, and using the same covering argument as in [17, Theorem 24.1] one obtains that there exist $\tau \in(0,1 / 2)$ and $C>0$ such that

$$
\mathcal{E}\left(0, \tau, e_{n}\right) \leqslant C\left(\int_{\partial E \cap B_{1}}\left|x_{n}-c\right|^{2} d \mathcal{H}^{n-1}+\Phi(0,1)+\Lambda r\right) .
$$

This inequality clearly implies (4.5).

While in our case the proofs for the height bound, the compactness, the reverse Poincaré and the Lipschitz approximation are exactly as in the case of $\Lambda$-minimizers of the perimeter, the next step in the regularity proof, i.e., the excess decay, is different. This is due to the interplay between the excess and the rescaled Dirichlet integral. We follow an argument similar to the one used in proving the flatness decay for the minimizers of the Mumford-Shah functional, see [3, Theorem 8.15]. To this aim we first prove the following weaker form of the Euler-Lagrange equation.

Proposition 4.9 Let $(E, u)$ be a $\Lambda$-minimizer of $\mathcal{f}$ in $B_{r}\left(x_{0}\right)$. For every vector field $X \in$ $C_{0}^{1}\left(B_{r}\left(x_{0}\right) ; \mathbb{R}^{n}\right)$ it holds

$$
\gamma \int_{\partial E} \operatorname{div}_{\tau} X d \mathcal{H}^{n-1}+\int_{\Omega} \sigma_{E}\left(|D u|^{2} \operatorname{div} X-2\langle D X D u, D u\rangle\right) d x \leqslant \Lambda \int_{\partial E}|X| d \mathcal{H}^{n-1},
$$

where $\operatorname{div}_{\tau}$ denotes the tangential divergence on $\partial E$.

Proof. The argument is similar to the one in [3, Theorem 7.35] and therefore we only give the sketch of the proof. For a given $X \in C_{0}^{1}\left(B_{r}\left(x_{0}\right) ; \mathbb{R}^{n}\right)$ we set for every small $t>0, \Phi_{t}(x)=x-t X(x)$, $E_{t}=\Phi_{t}(E)$ and $u_{t}(y)=u\left(\Phi_{t}^{-1}(y)\right)$. From the $\Lambda$-minimality it follows

$$
\gamma\left[P\left(E_{t}, B_{r}\left(x_{0}\right)\right)-P\left(E, B_{r}\left(x_{0}\right)\right)\right]+\int_{B_{r}\left(x_{0}\right)}\left(\sigma_{E_{t}}\left|D u_{t}\right|^{2}-\sigma_{E}|D u|^{2}\right) d x+\Lambda\left|E_{t} \Delta E\right| \geqslant 0 .
$$

The conclusion then follows from the same standard calculations used to derive the first variation of the perimeter and the Dirichlet integral (see [3, Theorem 7.35]) and observing that

$$
\lim _{t \rightarrow 0} \frac{\left|E_{t} \Delta E\right|}{t} \leqslant \int_{\partial E}\left|X \cdot v_{E}\right| d \mathcal{H}^{n-1},
$$

see for instance [11, Theorem 3.2].

We are ready to prove the excess improvement.

Proposition 4.10 Let $(E, u)$ be a $\Lambda$-minimizer of $\mathcal{F}$ in $B_{r}\left(x_{0}\right)$. For every $\tau \in(0,1 / 2)$ and $M$ there exists $\varepsilon_{5}=\varepsilon_{5}(\tau, M) \in(0,1)$ such that if $x_{0} \in \partial E$ and

$$
\varepsilon\left(x_{0}, r\right) \leqslant \varepsilon_{5} \quad \text { and } \quad \Phi\left(x_{0}, r\right)+r \leqslant M \varepsilon\left(x_{0}, r\right)
$$

then

$$
\varepsilon\left(x_{0}, \tau r\right) \leqslant C_{5}\left(\tau^{2} \varepsilon\left(x_{0}, r\right)+\Phi\left(x_{0}, 2 \tau r\right)+\tau r\right)
$$

for some constant $C_{5}$ depending on $\mathcal{F}\left(E, u ; B_{r}\left(x_{0}\right)\right)$. 
Proof. Without loss of generality we may assume that $\tau<1 / 8$. We argue by contradiction. After performing the same translation and rescaling used in the proof of Lemma 4.2, we may assume that there exist an infinitesimal sequence $\varepsilon_{h}$, a sequence $r_{h} \in \mathbb{R}$ and a sequence $\left(E_{h}, u_{h}\right)$ of $\Lambda r_{h^{-}}$ minimizers of $\mathcal{F}$ in $B_{1}$, with equibounded energies such that, denoting by $\varepsilon_{h}$ the excess of $E_{h}$ and by $\oiint_{h}$ the rescaled Dirichlet integral of $u_{h}$, we have

$$
\varepsilon_{h}(0,1):=\varepsilon_{h}, \quad \Phi_{h}(0,1)+r_{h} \leqslant M \varepsilon_{h},
$$

and

$$
\varepsilon_{h}(0, \tau)>C_{5}\left(\tau^{2} \varepsilon_{h}(0,1)+\Phi_{h}(0,2 \tau)+\tau r_{h}\right)
$$

Moreover, up to rotating each $E_{h}$ if necessary we may also assume, that for all $h$

$$
\varepsilon_{h}(0,1)=\varepsilon_{h}\left(0,1, e_{n}\right)=\frac{1}{2} \int_{\partial E_{h} \cap B_{1}}\left|v_{E_{h}}(x)-e_{n}\right|^{2} d \mathcal{H}^{n-1} .
$$

Step 1. Recalling Proposition 4.7, we have that for every $h$ sufficiently large there exists a 1 Lipschitz function $f_{h}: \mathbb{R}^{n-1} \rightarrow \mathbb{R}$ such that

$$
\sup _{\mathbb{R}^{n-1}}\left|f_{h}\right| \leqslant \varepsilon_{h}^{\frac{1}{2(n-1)}}, \quad \mathcal{H}^{n-1}\left(\partial E_{h} \Delta \Gamma_{f_{h}} \cap B_{1 / 2}\right) \leqslant C_{3} \varepsilon_{h}, \quad \int_{B_{1 / 2}^{n-1}}\left|D f_{h}\right|^{2} d x^{\prime} \leqslant C_{3} \varepsilon_{h} .
$$

Therefore, setting

$$
g_{h}\left(x^{\prime}\right):=\frac{f_{h}\left(x^{\prime}\right)-a_{h}}{\sqrt{\varepsilon_{h}}}, \quad \text { where } a_{h}=f_{B_{1 / 2}^{n-1}} f_{h} d x^{\prime}
$$

we may assume, up to a not relabeled subsequence, that the functions $g_{h}$ converge weakly in $H^{1}\left(B_{1 / 2}^{n-1}\right)$ and strongly in $L^{2}\left(B_{1 / 2}^{n-1}\right)$ to a function $g$.

We claim that $g$ is harmonic in $B_{1 / 2}$. To prove this it is enough to show that for any $\varphi \in$ $C_{0}^{1}\left(B_{1 / 2}^{n-1}\right)$

$$
\lim _{h \rightarrow \infty} \frac{1}{\sqrt{\varepsilon_{h}}} \int_{B_{1 / 2}^{n-1}} D f_{h} \cdot D \varphi d x^{\prime}=0 .
$$

In order to prove this equality we fix $\delta>0$ so that $\operatorname{supp} \varphi \times[-2 \delta, 2 \delta] \subset B_{1 / 2}$, choose a cut-off function $\psi: \mathbb{R} \rightarrow[0,1]$ with support in $(-2 \delta, 2 \delta)$ and $\psi \equiv 1$ in $[-\delta, \delta]$, and apply to $E_{h}$ the weak Euler-Lagrange equation (4.6) with $X=(0, \ldots, 0, \varphi \psi)$. By the height bound (Lemma 4.5) for $h$ large it holds $\partial E_{h} \cap B_{1 / 2} \subset B_{1 / 2}^{n-1} \times(-\delta, \delta)$. Therefore by denoting $v_{E_{h}}^{\prime}$ the vector made up by the first $n-1$ components of $v_{E_{h}}$, we have

$$
\begin{array}{r}
-\gamma \int_{\partial E_{h} \cap B_{1 / 2}} v_{E_{h}} \cdot e_{n} D \varphi \cdot v_{E_{h}}^{\prime} d \mathcal{H}^{n-1}+\int_{B_{1 / 2}} \sigma_{E_{h}}\left(\left|D u_{h}\right|^{2} \varphi \psi^{\prime}-2 D_{n} u_{h} D u_{h} \cdot D(\varphi \psi)\right) d x \\
\leqslant \Lambda r_{h} \int_{\partial E_{h} \cap B_{1 / 2}}|\varphi \psi| d \mathcal{H}^{n-1} .
\end{array}
$$

Thus, using the energy upper bound and recalling the inequality in (4.7), we have

$$
-\gamma \int_{\partial E_{h} \cap B_{1 / 2}} v_{E_{h}} \cdot e_{n} D \varphi \cdot v_{E_{h}}^{\prime} d \mathcal{H}^{n-1} \leqslant C \varepsilon_{h}
$$


for some constant $C$ depending on $\Lambda, \varphi$ and $\psi$, but independent of $h$. Therefore, dividing the above inequality by $\sqrt{\varepsilon_{h}}$, letting $h \rightarrow \infty$ and replacing $\varphi$ by $-\varphi$ we conclude that

$$
\lim _{h \rightarrow \infty} \frac{1}{\sqrt{\varepsilon_{h}}} \int_{\partial E_{h} \cap B_{1 / 2}} v_{E_{h}} \cdot e_{n} D \varphi \cdot v_{E_{h}}^{\prime} d \mathcal{H}^{n-1}=0
$$

From this equation we get (4.10) by observing that

$$
\begin{aligned}
& -\int_{\partial E_{h} \cap B_{1 / 2}} v_{E_{h}} \cdot e_{n} D \varphi \cdot v_{E_{h}}^{\prime} d \mathcal{H}^{n-1} \\
& =-\int_{\Gamma_{f_{h}} \cap B_{1 / 2}} v_{E_{h}} \cdot e_{n} D \varphi \cdot v_{E_{h}}^{\prime} d \mathcal{H}^{n-1}-\int_{\left(\partial E_{h} \backslash \Gamma_{f_{h}}\right) \cap B_{1 / 2}} v_{E_{h}} \cdot e_{n} D \varphi \cdot v_{E_{h}}^{\prime} d \mathcal{H}^{n-1} \\
& \quad+\int_{\left(\Gamma_{\left.f_{h} \backslash \partial E_{h}\right) \cap B_{1 / 2}} v_{E_{h}} \cdot e_{n} D \varphi \cdot v_{E_{h}}^{\prime} d \mathcal{H}^{n-1} .\right.}
\end{aligned}
$$

Indeed, recalling the second inequality in (4.9), we have that

$$
0=\lim _{h \rightarrow \infty} \frac{-1}{\sqrt{\varepsilon_{h}}} \int_{\Gamma_{f_{h}} \cap B_{1 / 2}} v_{E_{h}} \cdot e_{n} D \varphi \cdot v_{E_{h}}^{\prime} d \mathcal{H}^{n-1}=\lim _{h \rightarrow \infty} \frac{1}{\sqrt{\varepsilon_{h}}} \int_{B_{1 / 2}^{n-1}} \frac{D f_{h} \cdot D \varphi}{\sqrt{1+\left|D f_{h}\right|^{2}}} d x^{\prime},
$$

from which (4.10) immediately follows using the third inequality in (4.9).

Step 2. Since $g$ is harmonic we have for $\tau \in(0,1 / 8)$ that

$$
\begin{aligned}
\int_{B_{2 \tau}^{n-1}}\left|g\left(x^{\prime}\right)-g(0)-D g(0) \cdot x^{\prime}\right|^{2} d x^{\prime} & \leqslant c(n) \tau^{n+3} \sup _{B_{1 / 4}\left|D^{2} g\right|^{2}} \\
& \leqslant c(n) \tau^{n+3} \int_{B_{1 / 2}^{n-1}}|D g|^{2} d x^{\prime} .
\end{aligned}
$$

Since by (4.9) we have that

$$
\int_{B_{1 / 2}^{n-1}}|D g|^{2} d x \leqslant \liminf _{h \rightarrow \infty} \int_{B_{1 / 2}^{n-1}}\left|D g_{h}\right|^{2} d x \leqslant C_{3},
$$

and by the mean value property $(g)_{r}:=f_{B_{r}^{n-1}} g d x^{\prime}=g(0)$ and $(D g)_{r}=D g(0)$, we may conclude that

$$
\begin{aligned}
\lim _{h \rightarrow \infty} \int_{B_{2 \tau}^{n-1}}\left|g_{h}\left(x^{\prime}\right)-\left(g_{h}\right)_{2 \tau}-\left(D g_{h}\right)_{2 \tau} \cdot x^{\prime}\right|^{2} d x^{\prime} & \\
& =\int_{B_{2 \tau}^{n-1}}\left|g\left(x^{\prime}\right)-(g)_{2 \tau}-(D g)_{2 \tau} \cdot x^{\prime}\right|^{2} d x^{\prime} \leqslant \widehat{C} \tau^{n+3},
\end{aligned}
$$

for some constant $\widehat{C}$ depending only on $C_{3}$ and $n$. In turn, recalling the definition of $g_{h}$, this inequality is equivalent to

$$
\lim _{h \rightarrow \infty} \frac{1}{\varepsilon_{h}} \int_{B_{2 \tau}^{n-1}}\left|f_{h}\left(x^{\prime}\right)-\left(f_{h}\right)_{2 \tau}-\left(D f_{h}\right)_{2 \tau} \cdot x^{\prime}\right|^{2} d x^{\prime} \leqslant \widehat{C} \tau^{n+3}
$$


From this inequality, recalling that $\left|D f_{h}\right| \leqslant 1$ and setting

$$
c_{h}:=\frac{\left(f_{h}\right)_{2 \tau}}{\sqrt{1+\left|\left(D f_{h}\right)_{2 \tau}\right|^{2}}}, \quad v_{h}=\frac{\left(-\left(D f_{h}\right)_{2 \tau}, 1\right)}{\sqrt{1+\left|\left(D f_{h}\right)_{2 \tau}\right|^{2}}},
$$

we easily have

$$
\begin{aligned}
\limsup _{h \rightarrow \infty} \frac{1}{\varepsilon_{h}} \int_{\partial E_{h} \cap \Gamma_{f_{h}} \cap B_{2 \tau}} & \left|v_{h} \cdot x-c_{h}\right|^{2} d \mathcal{H}^{n-1} \\
& \leqslant \lim _{h \rightarrow \infty} \frac{1}{\varepsilon_{h}} \int_{B_{2 \tau}^{n-1}}\left|f_{h}\left(x^{\prime}\right)-\left(f_{h}\right)_{2 \tau}-\left(D f_{h}\right)_{2 \tau} \cdot x^{\prime}\right|^{2} d x^{\prime} \leqslant \widehat{C} \tau^{n+3} .
\end{aligned}
$$

On the other hand, arguing as in Step 1, we immediately get from the height bound and from the first two inequalities in (4.9) that

$$
\lim _{h \rightarrow \infty} \frac{1}{\varepsilon_{h}} \int_{\left(\partial E_{h} \backslash \Gamma_{f_{h}}\right) \cap B_{2 \tau}}\left|v_{h} \cdot x-c_{h}\right|^{2} d \mathcal{H}^{n-1}=0 .
$$

Hence, we conclude that

$$
\limsup _{h \rightarrow \infty} \frac{1}{\varepsilon_{h}} \int_{\partial E_{h} \cap B_{2 \tau}}\left|v_{h} \cdot x-c_{h}\right|^{2} d \mathcal{H}^{n-1} \leqslant \widehat{C} \tau^{n+3} .
$$

Note that

$$
\begin{aligned}
\int_{\partial E_{h} \cap B_{2 \tau}}\left|v_{E_{h}}-v_{h}\right|^{2} d \mathcal{H}^{n-1} & \leqslant 2 \int_{\partial E_{h} \cap B_{2 \tau}}\left|v_{E_{h}}-e_{h}\right|^{2} d \mathcal{H}^{n-1}+2\left|e_{n}-v_{h}\right|^{2} \mathcal{H}^{n-1}\left(\partial E_{h} \cap B_{2 \tau}\right) \\
& \leqslant 4 \varepsilon_{h}+C \int_{B_{1 / 2}^{n-1}}\left|D f_{h}\right|^{2} d x^{\prime} \leqslant C \varepsilon_{h}
\end{aligned}
$$

by the third inequality from (4.9). In particular, this shows that $\varepsilon_{h}\left(0,2 \tau, v_{h}\right) \rightarrow 0$ as $h \rightarrow \infty$. Therefore applying Theorem 4.8 and (4.11) we have for $h$ large that

$$
\varepsilon_{h}(0, \tau) \leqslant \varepsilon_{h}\left(0, \tau, v_{h}\right) \leqslant C_{4}\left(\hat{C} \tau^{2} \varepsilon_{h}(0,1)+\Phi_{h}(0,2 \tau)+2 \tau r_{h}\right)
$$

which is a contradiction to (4.8) if we choose $C_{5}>C_{4} \max \{\hat{C}, 2\}$.

Finally we give the proof of the regularity theorem (Theorem 1.2).

Proof of Theorem 1.2.

Step 1 . We begin by proving that for every $\tau \in(0,1)$ there exists $\varepsilon_{6}=\varepsilon_{6}(\tau)>0$ such that if $\varepsilon(x, r) \leqslant \varepsilon_{6}$ then

$$
Ð(x, \tau r) \leqslant C_{0} \tau \Phi(x, r)
$$

where $C_{0}$ is from Proposition 2.4. We argue by contradiction. After performing the same translation and rescaling used in the proof of Lemma 4.2 we may assume that there exist sequences $\varepsilon_{h}, r_{h}>0$ and a sequence $E_{h}$ of $\Lambda r_{h}$-minimizers of $\mathcal{F}$ in $B_{1}$ with equibounded energies, such that, denoting by $\varepsilon_{h}$ the excess of $E_{h}$ and by $\Phi_{h}$ the rescaled Dirichlet integral of $u_{E_{h}}$, we have that $0 \in \partial E_{h}$,

$$
\varepsilon_{h}(0,1)=\varepsilon_{h} \rightarrow 0 \quad \text { and } \quad \Phi_{h}(0, \tau)>C_{0} \tau \oiint_{h}(0,1) .
$$


By the energy upper bound (Theorem 4.3) and the compactness lemma (Lemma 4.6) we may assume that $E_{h} \rightarrow E$ in $L^{1}\left(B_{1}\right)$ and $0 \in \partial E$. Moreover by the lower semicontinuity of the excess we have that $\varepsilon(0,1)=0$ where $\varepsilon(0,1)$ is the excess of $E$ at 0 . Thus it follows that $E$ is a half space, say $H$, in $B_{1}$, see [17, Proposition 22.2]. In particular, for $h$ large it holds

$$
\left|\left(E_{h} \Delta H\right) \cap B_{1}\right|<\varepsilon_{0}(\tau)\left|B_{1}\right|
$$

where $\varepsilon_{0}$ is from Proposition 2.4 which gives a contradiction with the inequality in (4.12).

Step 2. Let $U \subset \subset \Omega$ be an open set. We show that for every $\tau \in(0,1)$ there exists $\varepsilon=\varepsilon(\tau, U)>$ 0 such that if $x_{0} \in \partial E, B_{r}\left(x_{0}\right) \subset U$ and $\mathcal{E}\left(x_{0}, r\right)+Ð\left(x_{0}, r\right)+r<\varepsilon$ then

$$
\varepsilon\left(x_{0}, \tau r\right)+\Phi\left(x_{0}, \tau r\right)+\tau r \leqslant C_{6} \tau\left(\varepsilon\left(x_{0}, r\right)+\Phi\left(x_{0}, r\right)+r\right) .
$$

First of all, if $\varepsilon \leqslant \varepsilon_{6}(\tau)$, then Step 1 implies

$$
\Phi\left(x_{0}, \tau r\right) \leqslant C_{0} \tau \Phi\left(x_{0}, r\right) .
$$

In order to prove (4.13) we may assume that $\tau<1 / 2$. Assume first that $D\left(x_{0}, r\right)+r \leqslant \tau^{-n} \varepsilon\left(x_{0}, r\right)$. Then if $\varepsilon\left(x_{0}, r\right)<\min \left\{\varepsilon_{5}(\tau, M), \varepsilon_{6}(2 \tau)\right\}$, for $M=\tau^{-n}$, it follows from Proposition 4.10 that

$$
\begin{aligned}
\varepsilon\left(x_{0}, \tau r\right) & \leqslant C_{5}\left(\tau^{2} \varepsilon\left(x_{0}, r\right)+\Phi\left(x_{0}, 2 \tau r\right)+\tau r\right) \\
& \leqslant C_{5}\left(\tau^{2} \varepsilon\left(x_{0}, r\right)+2 C_{0} \tau \Phi\left(x_{0}, r\right)+\tau r\right)
\end{aligned}
$$

where the last inequality follows from (4.14) applied to $2 \tau$. On the other hand if $\varepsilon\left(x_{0}, r\right) \leqslant$ $\tau^{n}\left(D\left(x_{0}, r\right)+r\right)$ we immediately obtain

$$
\varepsilon\left(x_{0}, \tau r\right) \leqslant \tau^{1-n} \varepsilon\left(x_{0}, r\right) \leqslant \tau\left(\Phi\left(x_{0}, r\right)+r\right) .
$$

Therefore (4.14) implies (4.13) by choosing $\varepsilon=\min \left\{\varepsilon_{5}(\tau, M), \varepsilon_{6}(2 \tau), \varepsilon_{6}(\tau)\right\}$.

Step 3. Let us fix $\sigma \in(0,1 / 2)$. We choose $\tau_{0} \in(0,1)$ such that $C_{6} \tau_{0} \leqslant \tau_{0}^{2 \sigma}$ where $C_{6}$ is the constant in (4.13). Let $U \subset \subset \Omega$ be an open set. We define

$\Gamma \cap U=\left\{x \in \partial E \cap U: \varepsilon(x, r)+\Phi(x, r)+r<\varepsilon\left(\tau_{0}, U\right)\right.$ for some $r>0$ such that $\left.B_{r}(x) \subset U\right\}$

where $\varepsilon\left(\tau_{0}\right)$ is from Step 2. Note that $\Gamma \cap U$ is relatively open in $\partial E$. We show that $\Gamma \cap U$ is $C^{1, \sigma}$-hypersurface.

Indeed (4.13) implies via standard iteration argument that if $x_{0} \in \Gamma \cap U$ there exist $r_{0}>0$ and a neighborhood $V$ of $x_{0}$ such that for every $x \in \partial E \cap V$ it holds

$$
\varepsilon\left(x, \tau_{0}^{k} r_{0}\right)+\Phi\left(x, \tau_{0}^{k} r_{0}\right)+\tau_{0}^{k} r_{0} \leqslant \tau_{0}^{2 \sigma k} \quad \text { for } k=0,1,2, \ldots
$$

In particular

$$
\varepsilon\left(x, \tau_{0}^{k} r_{0}\right) \leqslant \tau_{0}^{2 \sigma k}
$$

From this estimate and the density lower bound, arguing exactly as in [8, Theorem 8.2], we obtain that for every $x \in \partial E \cap V$ and $0<s<t<r_{0}$ it holds

$$
\left|\left(v_{E}\right)_{S}(x)-\left(v_{E}\right)_{t}(x)\right| \leqslant c t^{\sigma}
$$


for a constant $c$ depending on $\tau_{0}, r_{0}$ and $n$. Here

$$
\left(v_{E}\right)_{t}(x)=f_{\partial E \cap B_{t}(x)} v_{E}(y) d \mathcal{H}^{n-1} .
$$

The estimate (4.15) first implies that $\Gamma \cap U$ is $C^{1}$ (see for instance [8, Theorem 8.4]). By a standard argument we then deduce again from (4.15) that $\Gamma \cap U$ is $C^{1, \sigma}$-hypersurface. Finally we define $\Gamma:=\cup_{i}\left(\Gamma \cap U_{i}\right)$ where $\left(U_{i}\right)$ is an increasing sequence of open sets such that $U_{i} \subset \subset \Omega$ and $\Omega=\cup_{i} U_{i}$.

Step 4. Finally we prove that there exists $\eta>0$, depending only on $\frac{\beta}{\alpha}$ and $n$, such that

$$
\mathcal{H}^{n-1-\eta}(\partial E \backslash \Gamma)=0 .
$$

Since the argument is fairly standard we only give the sketch of the proof, see, e.g., Section 5 in [2], [4] and [5]. We set

$$
\Sigma=\left\{x \in \partial E \backslash \Gamma: \lim _{r \rightarrow 0} Ф(x, r)=0\right\} .
$$

Since by Lemma 2.2 $D u \in L_{l o c}^{2 p}(\Omega)$ for some $p>1$, depending only on $\frac{\beta}{\alpha}$ and $n$, we have that

$$
\operatorname{dim}_{\mathcal{H}}\left(\left\{x \in \Omega: \limsup _{r \rightarrow 0} \gg(x, r)>0\right\}\right) \leqslant n-p,
$$

where $\operatorname{dim}_{\mathcal{H}}$ denotes the Hausdorf dimension. The conclusion will follow if we show that $\Sigma=\emptyset$ when $n \leqslant 7$ and $\operatorname{dim}_{\mathcal{H}}(\Sigma) \leqslant n-8$ otherwise.

Let us first treat the case $n \leqslant 7$. We argue by contradiction and assume, up to a translation, that $0 \in \Sigma$. Let us take a sequence $r_{h} \rightarrow 0$ and set $E_{h}=\frac{E}{r_{h}}$ and $u_{h}(x)=r_{h}^{-1 / 2} u\left(r_{h} x\right)$. Then $\left(E_{h}, u_{h}\right)$ is $\Lambda r_{h}$-minimizer of $\mathcal{F}$. Since $D u_{h} \rightarrow 0$ in $L^{2}\left(B_{1}\right)$ Lemma 4.6 implies that, up to a subsequence, $E_{h}$ converges to a minimizer of the perimeter $E_{\infty}$ and moreover $\lim _{h \rightarrow \infty} P\left(E_{h}, U\right)=P\left(E_{\infty}, U\right)$ for every open set $U \subset B_{1}$ and $0 \in \partial E_{\infty}$. Since $n \leqslant 7$, we know that $\partial E_{\infty}$ is a smooth manifold. In particular, for any $\varepsilon>0$ there exists $r>0$ such that $\varepsilon\left(0, E_{\infty}, r\right)<\varepsilon$. However the above convergence of the perimeter implies that $\varepsilon\left(0, E_{h}, r_{h}\right)<\varepsilon$ when $h$ is large enough. By the definition of $\Gamma$ this contradicts the fact that $0 \in \Sigma$.

In the case $n \geqslant 8$ we claim that if $s>n-8$ then it holds $\mathcal{H}^{s}(\Sigma)=0$. The proof of this can be achieved arguing exactly as in the proof of [2, Theorem 5.6].

\section{Appendix}

We conclude by going through the proof of Lemma 2.2 and estimate all the relevant constants in the statement.

Proof of Lemma 2.2. We first prove the inequality of the lemma in the cube $Q=Q_{1}$. Denote $d(x)=\operatorname{dist}(x, \partial Q)$ and define

$$
\mathrm{C}_{k}=\left\{x \in Q \mid \frac{3}{4} 2^{-k-1} \leqslant d(x) \leqslant \frac{3}{4} 2^{-k}\right\} .
$$


Each $\mathrm{C}_{k}$ can be divided into cubes of side $\frac{3}{4} 2^{-k-1}$. We call this collection $G_{k}$. By Lemma 2.1 we have for $F(x)=d(x)^{n}|D u(x)|^{2}$ that

$$
f_{P} F d x \leqslant C_{0}\left(f_{\tilde{P}} F^{m} d x\right)^{\frac{1}{m}}
$$

where $\tilde{P}$ is the concentric cube to $P \subset \mathrm{C}_{k}$ or $P \subset Q_{1 / 4}$, for a constant $C_{0}=4^{n} C$, where $C$ is the constant from Lemma 2.1.

Denote next $\Phi_{t}=\{x \in Q \mid F(x)>t\}$, where $t>a:=f_{Q}|D u|^{2} d x$. Applying CalderónZygmund decomposition we obtain (in the proof [9, Lemma 6.2] choose $\lambda=2^{1 / m} C_{0}$ )

$$
\int_{\Phi_{t}} F d x \leqslant C_{1} t^{1-m} \int_{\Phi_{t}} F^{m} d x
$$

for

$$
C_{1}=2 \cdot 5^{n} 2^{n} \lambda=2 \cdot 2^{1 / m} 10^{n} C_{0} \leqslant 8 \cdot 40^{n} C=C_{S, n}^{2} 2^{11} \cdot 80^{n} \frac{\beta}{\alpha} .
$$

The result of [9, Proposition 6.1] now follows with the constants $A=C_{1}$ and $r=p>1$ such that

$$
C_{1}(p-1)=\frac{p-m}{2}
$$

that is

$$
p=\frac{2 C_{1}-m}{2 C_{1}-1}
$$

This leads to the inequality

$$
\int_{Q} F^{p} d x \leqslant 2 a^{p-1} \int_{Q} F d x
$$

for $a=f_{Q}|D u|^{2} d x$. Recalling the definition of $F$ we finally obtain

$$
f_{Q_{1 / 2}}|D u|^{2 p} d x \leqslant 2^{n+p n+1}\left(f_{Q}|D u|^{2} d x\right)^{p} .
$$

Let $B_{1} \subset \subset \Omega$. Observe that for any integer $h>1, Q_{1 / 2}$ can be covered by $h^{n}$ cubes of side length $1 / h$. Hence, $B_{1 / 2}$ can be covered by $N_{h}$ cubes $Q_{1 / 2 h}\left(x_{i}\right)$ having non-empty intersection with $B_{1 / 2}$ and $N_{h} \leqslant h^{n}$. Using the rescaled analogue of the inequality (5.1) we get

$$
\begin{aligned}
f_{B_{1 / 2}}|D u|^{2 p} d x & \leqslant \frac{2^{n}}{\omega_{n} h^{n}} \sum_{i=1}^{N_{h}} f_{Q_{1 / 2 h}\left(x_{i}\right)}|D u|^{2 p} d x \\
& \leqslant 2^{n+p n+1} \frac{2^{n}}{\omega_{n} h^{n}} \sum_{i=1}^{N_{h}}\left(f_{Q_{1 / h}\left(x_{i}\right)}|D u|^{2} d x\right)^{p} \\
& \leqslant 2^{n+p n+1} \frac{2^{n}}{\omega_{n} h^{n}} \sum_{i=1}^{N_{h}}\left(\frac{h^{n} \omega_{n}}{2^{n}} f_{B_{1}}|D u|^{2} d x\right)^{p}
\end{aligned}
$$


provided $h>4 \sqrt{n}$, in which case $Q_{1 / h}\left(x_{i}\right) \subset B_{1}$ for every $i=1, \ldots, N_{h}$. We may choose $h \leqslant 5 \sqrt{n}$ and thus we get

$$
f_{B_{1 / 2}}|D u|^{2 p} d x \leqslant 2^{2 n+1} 5^{n p} n^{n p / 2} \omega_{n}^{p-1}\left(f_{B_{1}}|D u|^{2} d x\right)^{p} .
$$

Acknowledgments. The research of NF was partially supported by the 2008 ERC Advanced Grant 226234 "Analytic Techniques for Geometric and Functional Inequalities" and by the FiDiPro project 2100002028. The research of VJ was supported by the Academy of Finland grant 268393. The authors want also to thank R. Kohn for pointing out to them the phenomenon of Taylor cones and G. De Philippis and A. Figalli for finding a mistake in the first version of the paper.

\section{REFERENCES}

1. Ambrosio, L. \& Buttazzo, G., An optimal design problem with perimeter penalization. Calc. Var. Partial Differential Equations 1 (1993), 55-69. Zbl0794. 49040 MR1261717

2. Ambrosio, L., Fusco, N. \& Hutchinson, J. E., Higher integrability of the gradient and dimension of the singular set for minimisers of the Mumford-Shah functional. Calc. Var. Partial Differential Equations 16 (2003), 187-215. Zbl1047.49015 MR1956854

3. Ambrosio, L., Fusco, N. \& Pallara, D., Functions of bounded variation and free discontinuity problems. Oxford University Press, New York, 2000. Zb10957.49001 MR1857292

4. De Lellis, C., Focardi, M. \& Ruffini, B., A note on the Hausdorff dimension of the singular set for minimizers of the Mumford-Shah energy. to appear in Adv. Calc. Var. 7 (2014), 539-545. Zbl1304. 49091 MR3276120

5. De Philippis, G. \& Figalli, A., Higher integrability for minimizers of the Mumford-Shah functional. Arch. Ration. Mech. Anal. 213 (2014), 491-502. Zbl06343911 MR3211857

6. De Philippis, G. \& Figalli, A., A note on the dimension of the singular set in free interface problems. Differential Integral Equations 28 (2015), 523-536.

7. Esposito, L. \& FusCO, N., A remark on a free interface problem with volume constraint. J. Convex. Anal. 18 (2011), 417-426. Zbl1216. 49033 MR2828497

8. GiUsti, E., Minimal surfaces and functions of bounded variation. Monographs in Mathematics, 80. Birkhäuser Verlag, Basel, 1984. Zb10545.49018 MR0775682

9. Giusti, E., Direct methods in the calculus of variations. World Scientific Publishing Co., Inc., River Edge, NJ, 2003. Zbl1028.49001 MR1962933

10. Goldman M., Novaga M. \& RufFini B., Existence and stability for a non-local isoperimetric model of charged liquid drops. Arch. Rational Mech. Anal. 217 (2015), 1-36.

11. Julin, V. \& Pisante, G., Minimality via second variation for microphase separation of diblock copolymer melts. to appear in J. Reine Angew. Math.

12. Larsen, C. J., Distance between components in optimal design problems with perimeter penalization. Ann. Scuola Norm. Sup. Pisa Cl. Sci. (4) 28 (1999), 641-649. Zbl0957 . 49028 MR1760534

13. LARSEN, C. J., Regularity of components in optimal design problems with perimeter penalization. Calc. Var. Partial Differential Equations 16 (2003), 17-29. Zbl1083.49031 MR1951490

14. Li, H., Halsey, T. \& LobKovsky, A., Singular shape of a fluid drop in an electric or magnetic field. Europhys. Lett. 27 (1994), 575-580.

15. Lin, F. H., Variational problems with free interfaces. Calc. Var. Partial Differential Equations 1 (1993), 149-168. Zb10794. 49038 MR1261721 
16. Lin, F. H. \& KoHn, R. V., Partial regularity for optimal design problems involving both bulk and surface energies. Chin. Ann. of Math. 20B (1999), 137-158. Zb10946. 49034 MR1699139

17. Maggi, F., Sets of finite perimeter and geometric variational problems. An introduction to geometric measure theory. Cambridge Studies in Advanced Mathematics, 135. Cambridge University Press, Cambridge, 2012. Zbl1255.49074 MR2976521

18. Ramos, A. \& Castellanos, A., Conical points in liquid-liquid interfaces subjected to electric fields. Phys. Lett. A 184 (1994), 268-272.

19. Stone, H. A., Lister, J. R. \& Brenner, M. P., Drops with conical ends in electric and magnetic fields. Proceedings: Mathematical, Physical and Engineering Sciences 455 (1999), 329-347. Zb10943. 76091

20. TAYlor, G.I. Disintegration of water drops in an electric field. Proc. R. Soc. Lond. A 280 (1964), 383397. Zb10119. 21101

21. ZelenY, J., Instability of electricfied liquid surfaces. Phys. Rev. 10 (1917), 1-6. 